\title{
Relative error accurate statistic based on nonparametric
}

\section{likelihood*}

\author{
Lorenzo Camponovo $^{\dagger}$ \\ Department of Business Economics, Health and Social Care \\ University of Applied Sciences and Arts of Italian Switzerland \\ Yukitoshi Matsushita ${ }^{\ddagger}$ \\ Taisuke Otsu ${ }^{\S}$ \\ Faculty of Economics \\ Department of Economics \\ Hitotsubashi University London School of Economics
}

October 28, 2020

\begin{abstract}
This paper develops a new test statistic for parameters defined by moment conditions that exhibits desirable relative error properties for the approximation of tail area probabilities. Our statistic, called the tilted exponential tilting (TET) statistic, is constructed by estimating certain cumulant generating functions under exponential tilting weights. We show that the asymptotic $p$-value of the TET statistic can provide an accurate approximation to the $p$ value of an infeasible saddlepoint statistic, which admits a Lugannani-Rice style adjustment with relative errors of order $n^{-1}$ both in normal and large deviation regions. Numerical results illustrate the accuracy of the proposed TET statistic. Our results cover both just- and over-identified moment condition models. A limitation of our analysis is that the theoretical approximation results are exclusively for the infeasible saddlepoint statistic, and closeness of the $p$-values for the infeasible statistic to the ones for the feasible TET statistic is only numerically assessed.
\end{abstract}

${ }^{*}$ The authors are grateful for helpful discussions with Elvezio Ronchetti and Richard Spady and comments by seminar participants at the University of Geneva. The authors also acknowledge helpful comments from the Editor, a Co-Editor, and anonymous referees. Financial supports from the JSPS KAKENHI (18K01541) (Matsushita) and the ERC Consolidator Grant (SNP 615882) (Otsu) are gratefully acknowledged.

${ }^{\dagger}$ E-mail: lorenzo.camponovo@supsi.ch. Address: University of Applied Sciences and Arts of Italian Switzerland, Galleria 2 - 100 Via Cantonale 6928, Manno, CH.

${ }^{\ddagger}$ E-mail: matsushita.y@r.hit-u.ac.jp. Address: Graduate School of Economics, Hitotsubashi University, 2-1 Naka, Kunitachi, Tokyo 186-8601, Japan.

${ }^{\S}$ E-mail: t.otsu@lse.ac.uk. Address: Department of Economics, London School of Economics, Houghton Street, London, WC2A 2AE, UK. 


\section{Introduction}

This paper is concerned with inference on moment condition models and proposes a new test statistic that shows desirable tail behaviors in terms of relative errors. For this problem, there are various test statistics available in the literature, such as the Wald, empirical likelihood (Owen, 1988), exponential tilting (Efron, 1981, 1982, Kitamura and Stutzer, 1997, and Imbens, Spady and Johnson, 1998), power divergence (Baggerly, 1998), and saddlepoint statistics (Robinson, Ronchetti and Young, 2003, and Ma and Ronchetti, 2011), among others. In particular, it is known that the empirical likelihood statistic admits the Bartlett correction, a higher-order refinement for the absolute error of the type I error probability (DiCiccio, Hall and Romano, 1991). This refinement in the absolute error is typically not achieved by other statistics, such as exponential tilting (Jing and Wood, 1996, and Baggerly, 1998).

For statistical inference, researchers are commonly interested in the accuracy of approximations for tail area probabilities or $p$-values of test statistics. For this purpose, the relative error rather than the absolute one delivers a more relevant measure of accuracy, and various procedures typically based on saddlepoint approximations are developed (Tingley and Field, 1990, Daniels and Young, 1991, Jing and Robinson, 1994, Robinson, Ronchetti and Young, 2003, and Kolassa and Robinson, 2011, among others). In particular, Robinson, Ronchetti and Young (2003) considered the situation where the cumulant generating function is known to the researcher and developed a novel saddlepoint statistic that is asymptotically chi-squared distributed with a relative error of order $n^{-1}$ even in the large deviation region. Although this statistic is generally infeasible due to the requirement on the knowledge of the cumulant generating function, Robinson, Ronchetti and Young (2003) and Ma and Ronchetti (2011) proposed some feasible versions of the saddlepoint statistic by using the exponential tilting weights (Efron, 1981, 1982).

The basic idea of our statistic is to note that the conventional exponential tilting statistic is constructed from estimating the cumulant generating function by the sample average, and to modify the cumulant estimation by using the exponential tilting weights instead of the uniform weights. In other words, we tilt the exponential tilting statistic. Thus, the new statistic is called the tilted exponential tilting (TET) statistic.

We show that the TET statistic is asymptotically chi-squared distributed, and demonstrate that its asymptotic $p$-value provides an accurate approximation to the $p$-value of some ideal (but infeasible) saddlepoint statistic, which admits a Lugannani-Rice style adjustment with a relative error of order $n^{-1}$ both in normal and large deviation regions. Thus, as far as the $p$-value for the TET statistic is well approximated by the one for the ideal statistic, we can 
argue that the asymptotic $p$-value approximation for the TET statistic using the chi-squared distribution is very accurate even in the tails. Due to technical difficulty, however, this paper only establishes the theoretical approximation results for the ideal saddlepoint statistic, and admittedly the approximation property between the ideal saddlepoint and feasible TET statistics is only numerically investigated. We note that both the TET and ideal statistics are new in the literature, and different from the saddlepoint statistics discussed above. Furthermore, our results on the TET statistic cover both just- and over-identified moment condition models.

We emphasize that accuracy of $p$-values in tail areas for testing moment condition models is a substantial issue in applied research. In practice, researchers are concerned with accuracy of relatively small $p$-values (say, less than $10 \%$ ) which will be typical borderlines to decide whether the null should be rejected or not. As our simulation studies below indicate, the empirical quantiles of the TET statistic are very close to the ones of the limiting chi-squared distribution for the tail areas. Furthermore, we note that moment condition models are widely used in empirical economic analyses, and it is well known that the (first-order) asymptotic approximations for those models may not provide accurate inference methods in small to moderate samples (see, e.g., the special issue in the Journal of Business \& Economic Statistics, vol. 14).

Finally, we study through Monte Carlo simulations the accuracy of the proposed TET statistic. In addition to a benchmark setup for inference on means, we consider both just- and over-identified instrumental variable regression models. The numerical results highlight a desirable accuracy of our test statistic. In particular, the empirical quantiles of the TET statistic are extremely close to those of the limiting distribution even for small sample sizes.

This paper is organized as follows. In Section 2, we consider a benchmark case, simple parameter hypothesis testing for just-identified moment conditions, and present basic theoretical results illustrated by a small simulation study. Section 3 extends our method to testing problems for composite hypotheses (Section 3.1), and overidentifying restrictions (Section 3.2). Finally, Section 4 presents simulation results for the general case. All proofs are presented in Appendix A. Appendix B contains figures for simulation results in Section 4.

\section{Benchmark case}

In this section, we present the basic idea of the new test statistic and its theoretical and numerical properties under a benchmark setup. Section 2.1 introduces our TET statistic. Section 2.2 illustrates its finite sample accuracy through Monte Carlo simulation. In Section 2.3, we discuss that the TET statistic has desirable relative error properties for the approximation of tail area 
probabilities.

\subsection{Tilted exponential tilting statistic}

Suppose we observe an i.i.d. sample $\left\{X_{i}\right\}_{i=1}^{n}$ of $X$. Let $F$ be the distribution function of $X$ and $\mathbb{E}[\cdot]$ be the expectation under $F$. Consider the (just-identified) moment conditions

$$
\mathbb{E}\left[g\left(X, \theta_{0}\right)\right]=0,
$$

where $g$ is a $p$-dimensional vector of moment functions and $\theta_{0}$ is a $p$-dimensional vector of true parameter values. In this section, we focus on hypothesis testing for the null $H_{0}: \theta_{0}=0$ against the two-sided alternative $H_{1}: \theta_{0} \neq 0$. Since we do not specify the parametric distribution form of $X$, testing methods based on parametric likelihood theory, such as the likelihood ratio and score tests, are not applicable. However, there are several ways to test $H_{0}$ in this setting. For example, we can implement the Wald test based on some estimator of $\theta_{0}$. Also based on some nonparametric likelihood, we can conduct likelihood ratio or score type tests (see, e.g., Owen, 2001).

We propose a new test statistic for $H_{0}$, which exhibits a desirable finite sample accuracy. Our test statistic is constructed by evaluating the exponential tilting statistic (Efron, 1981, 1982, Kitamura and Stutzer, 1997, and Imbens, Spady and Johnson, 1998) under the exponential tilting weights based on the restriction $\mathbb{E}\left[g\left(X, \theta_{0}\right)\right]=0$ under the null $H_{0}: \theta_{0}=0$. Let $\hat{\lambda}$ be a solution of $\sum_{i=1}^{n} e^{\hat{\lambda}^{\prime} g\left(X_{i}, 0\right)} g\left(X_{i}, 0\right)=0$. The conventional exponential tilting statistic for $H_{0}: \theta_{0}=0$ is written as

$$
T_{n}^{\mathrm{et}}=-2 \log \left(\frac{1}{n} \sum_{i=1}^{n} e^{\hat{\lambda}^{\prime} g\left(X_{i}, 0\right)}\right)
$$

It is known that $n T_{n}^{\text {et }}$ converges in distribution to the $\chi_{p}^{2}$ distribution under $H_{0}$. This statistic is obtained by minimization of the empirical relative entropy

$$
\min _{\pi_{1}, \ldots, \pi_{n}} \sum_{i=1}^{n} n \pi_{i} \log \left(n \pi_{i}\right), \quad \text { s.t. } \sum_{i=1}^{n} \pi_{i} g\left(X_{i}, 0\right)=0, \quad \sum_{i=1}^{n} \pi_{i}=1 .
$$

By applying the Lagrange multiplier method, the solution is written as

$$
\hat{\pi}_{i}=\frac{e^{\hat{\lambda}^{\prime} g\left(X_{i}, 0\right)}}{\sum_{j=1}^{n} e^{\hat{\lambda}^{\prime} g\left(X_{j}, 0\right)}},
$$

for $i=1, \ldots, n$. Note that by construction these optimal weights are positive and satisfy the moment condition $\sum_{i=1}^{n} \hat{\pi}_{i} g\left(X_{i}, 0\right)=0$. Indeed, the empirical distribution using the weights $\left\{\hat{\pi}_{i}\right\}_{i=1}^{n}$ 
is an asymptotically efficient estimator of the distribution function of $X$ under the restriction $\mathbb{E}\left[g\left(X, \theta_{0}\right)\right]=0$ subject to the null $H_{0}: \theta_{0}=0$ (Brown and Newey, 1998).

Intuitively, the exponential tilting statistic $T_{n}^{\text {et }}$ is constructed by taking expectation of $e^{\hat{\lambda}^{\prime} g\left(X_{i}, 0\right)}$ under the empirical distribution with weights $1 / n$. Our proposal is to replace the uniform weights by the optimal ones under $H_{0}$, and to take expectation of $e^{\hat{\lambda}^{\prime} g\left(X_{i}, 0\right)}$ under the tilted empirical distribution with weights $\left\{\hat{\pi}_{i}\right\}_{i=1}^{n}$, that is

$$
T_{n}^{\mathrm{tet}}=2 \log \left(\sum_{i=1}^{n} \hat{\pi}_{i} e^{\hat{\lambda}^{\prime} g\left(X_{i}, 0\right)}\right)=2\left[\log \left(\sum_{i=1}^{n} e^{2 \hat{\lambda}^{\prime} g\left(X_{i}, 0\right)}\right)-\log \left(\sum_{i=1}^{n} e^{\hat{\lambda}^{\prime} g\left(X_{i}, 0\right)}\right)\right] .
$$

We call this statistic the tilted exponential tilting (TET) statistic. As shown in the proof of Theorem 1, the reason for the positive sign of $T_{n}^{\text {tet }}$ can be seen from a second-order expansion around $\hat{\lambda}^{\prime} g\left(X_{i}, 0\right)=0$,

$$
n T_{n}^{\mathrm{tet}}=n \hat{\lambda}^{\prime}\left[\sum_{i=1}^{n} \hat{\pi}_{i} g\left(X_{i}, 0\right) g\left(X_{i}, 0\right)^{\prime}\right] \hat{\lambda}+o_{p}(1)
$$

under $H_{0}$, where we used $\sum_{i=1}^{n} \hat{\pi}_{i} g\left(X_{i}, 0\right)=0$. It will be shown that the right hand side converges in distribution to the $\chi_{p}^{2}$ distribution under $H_{0}$. To make the argument rigorous, we impose the following assumption.

Assumption 1. $\left\{X_{i}\right\}_{i=1}^{n}$ is i.i.d., $\mathbb{E}\left[\left|g\left(X, \theta_{0}\right)\right|^{\zeta}\right]<\infty$ for some $\zeta>2$, and $\mathbb{E}\left[g\left(X, \theta_{0}\right) g\left(X, \theta_{0}\right)^{\prime}\right]$ is nonsingular.

All conditions are standard. Based on these conditions, the limiting null distribution of the TET statistic is obtained as follows.

Theorem 1. Under Assumption 1 and $H_{0}: \theta_{0}=0$, the TET statistic $n T_{n}^{\text {tet }}$ converges in distribution to the $\chi_{p}^{2}$ distribution.

Therefore, under $H_{0}$, the TET statistic $n T_{n}^{\text {tet }}$ is asymptotically equivalent to the exponential tilting statistic $n T_{n}^{\text {et }}$. We can also show that they have the same local power function under local alternatives.

\subsection{Simulation for benchmark case}

To illustrate finite sample accuracy of the TET statistic, we provide a preliminary simulation result. We generate random samples $\left\{X_{i}\right\}_{i=1}^{n}$ of sizes $n=20,40$, and 80 according to $X_{i} \sim$ $N(0,1)$. The parameter $\theta_{0}$ is defined by the moment condition $\mathbb{E}\left[g\left(X, \theta_{0}\right)\right]=\mathbb{E}\left[X-\theta_{0}\right]=0$. 
We are interested in testing the null hypothesis $H_{0}: \theta_{0}=0$ against the alternative $H_{1}: \theta_{0} \neq 0$, and compare the proposed TET statistic $n T_{n}^{\text {tet }}$ with the exponential tilting statistic $n T_{n}^{\text {et }}$ and the conventional Wald statistic. All the statistics converge in distribution to the $\chi_{1}^{2}$ distribution under $H_{0}$. Figure 1 reports the q-q plots of the empirical quantiles of these test statistics against those of the $\chi_{1}^{2}$ distribution. The number of Monte Carlo replications is 20, 000 .

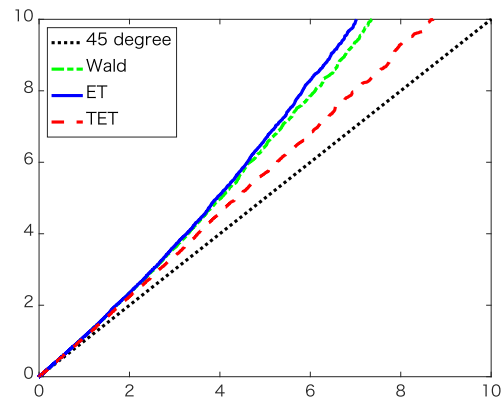

(a) $n=20$

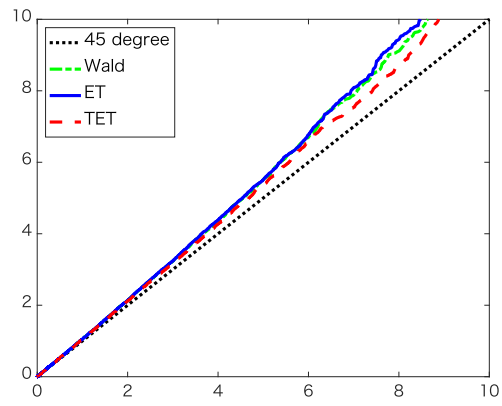

(b) $n=40$

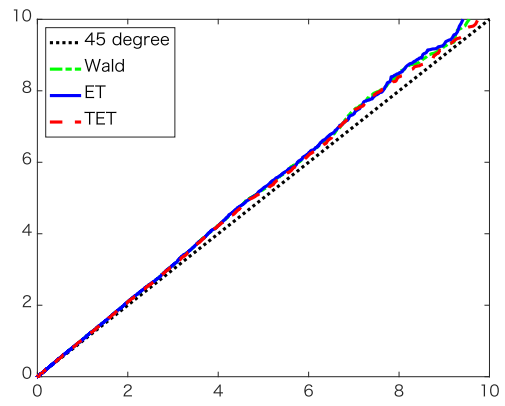

(c) $n=80$

Figure 1: Empirical quantiles of the TET statistic (dashed red line), exponential tilting statistic (solid blue line), and Wald statistic (dash-dotted green line) against quantiles of the $\chi_{1}^{2}$ distribution for (a) $n=20$, (b) $n=40$, and (c) $n=80$.

As this figure shows, the empirical quantiles of the TET statistic are closer to those of the limiting $\chi_{1}^{2}$ distribution even for relatively small sample sizes. The accuracies of the exponential tilting and Wald statistics increase as the sample size increases, although the TET statistic outperforms for all cases. In the next subsection, we provide some explanation for this excellent finite sample performance of the TET statistic.

\subsection{Relative error properties}

\subsubsection{Overview}

The main purpose of this paper is to provide some theoretical justification for the desirable accuracy of the TET statistic $n T_{n}^{\text {tet }}$ using $\chi^{2}$ asymptotic approximation particularly in the tail area as observed in the q-q plots in the last subsection. Based on the statistics literature of saddlepoint approximations (see, e.g., Jensen, 1995, for a review), an ideal goal for this purpose is to evaluate the order (say, $\varrho_{n}$ ) in the relative error for the $\chi^{2}$ asymptotic approximation:

$$
\operatorname{Pr}\left\{n T_{n}^{\text {tet }} \geq n t: F\right\}=\left\{1-F_{p}(n t)\right\}\left(1+O\left(\varrho_{n}\right)\right),
$$

and compare the approximation error $O\left(\varrho_{n}\right)$ with the ones for other statistics, where $F_{p}$ is the cumulative distribution function of the $\chi_{p}^{2}$ distribution. However, the existing techniques for 
saddlepoint approximations are mostly confined to smooth function models for finite dimensional sample means and parametric models, and it is difficult to establish the approximation result for the TET statistic as in (3) under moment condition models. To the best of our knowledge, there is no relative error approximation results for the GMM or generalized empirical likelihood-based statistic under general moment condition models.

To circumvent this technical difficulty, we consider an infeasible version of the TET statistic, called $T_{n}$ in (5) below, by replacing the weighted average in (2) using $\left\{\hat{\pi}_{i}\right\}_{i=1}^{n}$ with the population mean. Although $T_{n}$ is infeasible, it is possible to establish certain relative error approximation results for $\operatorname{Pr}\left\{n T_{n} \geq n t: F\right\}$ as in (3) (see, Theorem 2 below).

Based on these considerations, we assume that the $p$-value $p^{\text {tet }}=\operatorname{Pr}\left\{n T_{n}^{\text {tet }} \geq n t^{\text {tet,o }}: F\right\}$ for the TET statistic is well approximated by its infeasible version $p^{\text {ideal }}=\operatorname{Pr}\left\{n T_{n} \geq n t^{\circ}: F\right\}$, where $t^{\text {tet,o }}$ and $t^{\mathrm{o}}$ are observed values of $T_{n}^{\text {tet }}$ and $T_{n}$, respectively. Then, under this assumption, we concentrate on the relative error properties to approximate the $p$-value of the proxy $p^{\text {ideal }}$ for $p^{\text {tet }}$. Our main results (Theorems 2 and 3 below) establish the relation between $p^{\text {ideal }}$ and the asymptotic $p$-value $p^{\text {asy }}=1-F_{p}\left(n t^{\text {tet,oo }}\right)$ for our TET test as

$$
p^{\text {ideal }}=p^{\text {asy }}\left(1+r_{n}\left(\hat{\lambda}^{\mathrm{o}}\right)\right)\left(1+O\left(n^{-1}\right)\right),
$$

where $\hat{\lambda}^{\circ}$ is an observed value of $\hat{\lambda}$ and $r_{n}(\cdot)$ is a function defined in (19). Based on this relative error approximation, we argue that $r_{n}(\cdot)$ exhibits a desirable property not shared by the conventional exponential tilting test.

A major limitation of this paper is that due to technical difficulty in analyzing $T_{n}^{\text {tet }}$, we cannot provide any theory to characterize the quality of the approximation for $p^{\text {tet }}$ by $p^{\text {ideal }}$. The original object of interest, the ratio of the $p$-value $p^{\text {tet }}$ of the TET statistic to its asymptotic approximation $p^{\text {asy }}$, can be decomposed as

$$
\frac{p^{\text {tet }}}{p^{\text {asy }}}=\left(1+\rho_{1}\right)\left(1+\rho_{2}\right)
$$

where $\rho_{1}=\left(p^{\text {tet }}-p^{\text {ideal }}\right) / p^{\text {ideal }}$ and $\rho_{2}=\left(p^{\text {ideal }}-p^{\text {asy }}\right) / p^{\text {asy }}$. Our result in (4) only characterizes $\rho_{2}$. For the benchmark simulation in Section 2.2, we evaluate the magnitudes of the component $\rho_{1}$ by the ratios $\left|\frac{\rho_{1}}{\rho_{2}}\right|$ for 1000 Monte Carlo replications, and find that their interquartile ranges are: $[0.49,1.71]$ for $n=20,[0.53,1.47]$ for $n=40$, and $[0.57,1.45]$ for $n=80$. Thus, at least for the benchmark simulation setup, the approximation error $\rho_{1}$ is comparable to $\rho_{2}$, which will be characterized by our theorems. Theoretical analysis on $\rho_{1}$ is a substantial challenge and should 
be left for future research.

\subsubsection{Ideal statistic $T_{n}$}

We now introduce the ideal statistic $T_{n}$. In the definition of the TET statistic (2), we propose to take expectation of $e^{\hat{\lambda}^{\prime} g\left(X_{i}, 0\right)}$ under the tilted weights $\left\{\hat{\pi}_{i}\right\}_{i=1}^{n}$ satisfying $\sum_{i=1}^{n} \hat{\pi}_{i} g\left(X_{i}, 0\right)=0$. The ideal (but infeasible) statistic is given by evaluating this expectation under the population:

$$
T_{n}=2 K(\hat{\lambda})
$$

where $K(\lambda)=\log \mathbb{E}\left[e^{\lambda^{\prime} g(X, 0)}\right]$ is the cumulant generating function of $g(X, 0)$, and $\hat{\lambda}$ solves $\sum_{i=1}^{n} e^{\hat{\lambda}^{\prime} g\left(X_{i}, 0\right)} g\left(X_{i}, 0\right)=0$. Observe that $T_{n}$ is infeasible because it involves the expectation $\mathbb{E}[\cdot]$ to evaluate the cumulant. Furthermore, $T_{n}$ is different from the saddlepoint statistic proposed in Robinson, Ronchetti and Young (2003) because it does not involve any estimators of $\theta_{0}$.

To study the relative error property of $T_{n}$, we introduce further notation. Let $\breve{g}(x)=$ $\mathbb{E}\left[g(X, 0) g(X, 0)^{\prime}\right]^{-1 / 2} g(x, 0)$ be a normalized counterpart of $g(x, 0)$ so that $\operatorname{Var}(\check{g}(X))=I$, and define $\check{\lambda}=\mathbb{E}\left[g(X, 0) g(X, 0)^{\prime}\right]^{1 / 2} \hat{\lambda}$. Based on the definition of $\hat{\lambda}, \check{\lambda}$ can be written as a solution of $\sum_{i=1}^{n} \psi\left(X_{i}, \check{\lambda}\right)=0$, where $\psi(x, y)=-e^{y^{\prime} \check{g}(x)} \check{g}(x)$. Thus, $\psi(x, y)$ may be interpreted as an estimating function for $\check{\lambda}$. Also let $K(t, y)=\log \mathbb{E}\left[e^{t^{\prime} \psi(X, y)}\right]$ and $t(y)$ be a solution of $\frac{\partial K(t(y), y)}{\partial t}=0$. We impose the following high level assumption.

Assumption 2. The density $f_{\check{\lambda}}$ of $\check{\lambda}$ exists and has the saddlepoint approximation

$$
f_{\check{\lambda}}(y)=\left(\frac{n}{2 \pi}\right)^{p / 2} e^{-n h(y)} \frac{\operatorname{det} B(y)}{\sqrt{\operatorname{det} \Sigma(y)}}\left(1+O\left(n^{-1}\right)\right),
$$

where

$$
\begin{aligned}
h(y) & =K(t(y), y), \\
B(y) & =e^{n K(t(y), y)} \mathbb{E}\left[e^{t(y)^{\prime} \psi(X, y)} \frac{\partial \psi(X, y)}{\partial y}\right], \\
\Sigma(y) & =e^{n K(t(y), y)} \mathbb{E}\left[e^{t(y)^{\prime} \psi(X, y)} \psi(X, y) \psi(X, y)^{\prime}\right] .
\end{aligned}
$$

Assumption 2 requires existence of the usual form of the saddlepoint approximation of the density of $\check{\lambda}$. We note that the saddlepoint approximation error in (6) is typically of relative order $O\left(n^{-1}\right)$. For primitive conditions under which this assumption holds, we refer to Field (1982), Skovgaard (1990), Jensen and Wood (1998), and Almudevar, Field and Robinson (2000). 
For example, suppose $\mathbb{E}\left[g(X, 0) g(X, 0)^{\prime}\right]$ is invertible and all components in $\psi(x, y)$ and their first four derivatives with respect to $y$ are bounded and continuous as functions of $x$ at each $y$. Then certain smoothness conditions that enable the development of an Edgeworth expansion are sufficient to guarantee Assumption 2 (see, Ma and Ronchetti, 2011, pp. 154-155). ${ }^{1}{ }^{2}$

Let $F_{p}$ be the cumulative distribution function of the $\chi_{p}^{2}$ distribution. The relative error property for the approximation of the tail area probability of $T_{n}$ is established as follows.

Theorem 2. Suppose that Assumptions 1 and 2 hold. Then under $H_{0}: \theta_{0}=0$,

$$
\operatorname{Pr}\left\{n T_{n} \geq n t: F\right\}=\left\{1-F_{p}(n \xi(t))\right\}\left(1+O\left(n^{-1}\right)\right),
$$

uniformly over $t \in(0, \varepsilon)$ for some $\varepsilon>0$, where $\xi(t)=\left(\sqrt{t}-\frac{\log G(\sqrt{t})}{n \sqrt{t}}\right)^{2}$ and $G(\cdot)$ is defined in (17) in the Appendix.

Theorem 2 provides a Lugannani-Rice style accurate approximation formula for the tail area probability of $T_{n}$. This approximation holds not only for the normal region (i.e., $n t$ is bounded) but also for the large deviation region (i.e., $t$ is bounded). This theorem shows that the ideal statistic $T_{n}$ admits a relative error of order $O\left(n^{-1}\right)$ up to the large deviation region. Note that relative error orders typically deliver a more useful measure of the quality of tail area approximation compared to absolute ones. Robinson, Ronchetti and Young (2003) established an analogous desirable relative error property for their saddlepoint statistic, which is also infeasible in the present setup. Also, Huber and Ronchetti (2009, ch. 14.6) argued that for parametric models the three classical hypothesis tests (i.e., likelihood ratio, Wald, and score) do not satisfy the relative error property as in (7).

\footnotetext{
${ }^{1}$ More precisely, let $U^{y}=\left(U_{1}^{y \prime}, U_{2}^{y \prime}, U_{2}^{y \prime}\right)$, where $U_{1}^{y}=\psi(X, y), U_{2}^{y}$ is the vector formed by the elements of $\partial \psi(X, y) / \partial y^{\prime}$ and $\psi(X, y) \psi(X, y)^{\prime}$, and $U_{3}^{y}$ is the vector formed by the elements of $\partial U_{2}^{y} / \partial y^{\prime}$. Then let $F_{U}^{y}$ be the distribution function of $U^{y}$ and define a random vector $\tilde{U}^{y}$ with the distribution function

$$
F_{\tilde{U}}^{y}\left(u_{1}, u_{2}, u_{3}\right)=\int_{\left(a_{1}, a_{2}, a_{3}\right) \leq\left(u_{1}, u_{2}, u_{3}\right)} e^{t(y)^{\prime} a_{1}-K(t(y), y)} d F_{U}^{y}\left(a_{1}, a_{2}, a_{3}\right),
$$

and the characteristic function $\phi^{y}(\xi)=\mathbb{E}\left[e^{\mathrm{i} \xi^{\prime} \tilde{U}^{y}}\right]$. Based on this notation, the smoothness condition is stated as: for each $y$, there exist positive constants $c, C$, and $c_{1} \operatorname{such}$ that (i) $c<\operatorname{det}\left(\operatorname{Var}\left(\tilde{U}^{y}\right)\right)<C$, and (ii) $\left|\phi^{y}(\xi)\right| \leq 1-c_{1}$ for all $c<|\xi|<C n^{\left(\operatorname{dim} \tilde{U}^{y}+1\right) / 2}$.

${ }^{2}$ To be specific, consider the (just-identified) instrumental variable regression model, where $g\left(X, Y, Z, \theta_{0}\right)=$ $Z\left(Y-X^{\prime} \theta_{0}\right)$ for a dependent variable $Y$, endogenous regressors $X$, and instrumental variables $Z$. Assumptions 1 and 2 are satisfied if $\left\{X_{i}, Y_{i}, Z_{i}\right\}_{i=1}^{n}$ is i.i.d., $\mathbb{E}\left[g\left(X, Y, Z, \theta_{0}\right) g\left(X, Y, Z, \theta_{0}\right)^{\prime}\right]$ invertible, and the random variable $W=g\left(X, Y, Z, \theta_{0}\right)$ is continuously distributed on some compact support $\mathcal{W}$, which is typically guaranteed by the compact support of the observables $\left(Y, X^{\prime}, Z^{\prime}\right)$. Although this is a rather restrictive sufficient condition, our simulation studies in Sections 2.2 and 4 do not impose compact support for the data and we can still observe desirable performances of the TET statistic.

We note that most existing results using the saddlepoint approximation require finite moment generating functions (around zero) for the estimating equations (i.e., $\psi(X, y)$ in our notation). A notable exception is Jing, Shao and Zhou (2004) who established the saddlepoint approximation for tail probabilities of the t-statistic with no moment conditions. Although it is beyond the scope of this paper, it is interesting to see whether their theoretical development can be adapted to our context.
} 
The adjustment by the transform $\xi(t)$ to achieve relative error refinement is analogous to the one in Kolassa and Robinson (2011) for the (parametric) likelihood ratio statistic. In general, the function $G(\cdot)$ requires numerical integration over a sphere of dimension $p$, but a Monte Carlo approximation to any degree of accuracy required can be obtained; see Kolassa and Robinson (2011) for details.

\subsubsection{Relative error property of the TET statistic}

Motivated by the desirable relative error property of the ideal statistic $T_{n}$, we now establish the result in (4) and argue that the TET statistic $T_{n}^{\text {tet }}$ provides an accurate approximation to the tail area probabilities of the ideal statistic $T_{n}$. To this end, we introduce the function

$$
K^{\mathrm{tet}}(\lambda)=\log \left(\sum_{i=1}^{n} \hat{\pi}_{i}^{\mathrm{o}} e^{\lambda^{\prime} g\left(x_{i}^{\mathrm{o}}, 0\right)}\right),
$$

where $\left\{x_{i}^{o}\right\}_{i=1}^{n}$ and $\left\{\hat{\pi}_{i}^{\mathrm{o}}\right\}_{i=1}^{n}$ are the observed values of $\left\{X_{i}\right\}_{i=1}^{n}$ and $\left\{\hat{\pi}_{i}\right\}_{i=1}^{n}$, respectively. We can see that the observed values of $T_{n}$ and $T_{n}^{\text {tet }}$ are given by $t_{n}=2 K\left(\hat{\lambda}^{\mathrm{o}}\right)$ and $t_{n}^{\text {tet }}=2 K^{\text {tet }}\left(\hat{\lambda}^{\mathrm{o}}\right)$, respectively, where $\hat{\lambda}^{\mathrm{o}}$ is the observed value of $\hat{\lambda}$. Taylor expansions of $K(\lambda)$ and $K^{\text {tet }}(\lambda)$ around $\lambda=0$ yield

$$
\begin{aligned}
K(\lambda) & =\frac{1}{2} \lambda^{\prime} \mathbb{E}\left[g(X, 0) g(X, 0)^{\prime}\right] \lambda+O\left(|\lambda|^{3}\right), \\
K^{\mathrm{tet}}(\lambda) & =\frac{1}{2} \lambda^{\prime}\left(\sum_{i=1}^{n} \hat{\pi}_{i}^{\mathrm{o}} g\left(x_{i}^{\mathrm{o}}, 0\right) g\left(x_{i}^{\mathrm{o}}, 0\right)^{\prime}\right) \lambda+O\left(|\lambda|^{3}\right) .
\end{aligned}
$$

These expansions highlight some interesting analogies between $T_{n}$ and $T_{n}^{\text {tet }}$. By the argument in the proof of Theorem 1, the sample counterpart of the difference $K(\lambda)-K^{\text {tet }}(\lambda)$ is of order $O_{p}\left(n^{-1 / 2}|\lambda|^{2}\right)$.

On the other hand, if we consider an analogous function $K^{\mathrm{et}}(\lambda)=-\log \left(\frac{1}{n} \sum_{i=1}^{n} e^{\lambda^{\prime} g\left(x_{i}^{\mathrm{o}}, 0\right)}\right)$ for the exponential tilting statistic so that $t_{n}^{\text {et }}=2 K^{\text {et }}\left(\hat{\lambda}^{\mathrm{o}}\right)$, then an expansion yields

$$
K^{\mathrm{et}}(\lambda)=-\lambda^{\prime} \bar{g}^{\mathrm{o}}-\frac{1}{2} \lambda^{\prime} \hat{V}^{\mathrm{o}} \lambda+O\left(|\lambda|^{3}\right)
$$

where $\bar{g}^{\mathrm{o}}=\frac{1}{n} \sum_{i=1}^{n} g\left(x_{i}^{\mathrm{o}}, 0\right)$ and $\hat{V}^{\mathrm{o}}=\frac{1}{n} \sum_{i=1}^{n}\left(g\left(x_{i}^{\mathrm{o}}, 0\right)-\bar{g}^{\mathrm{o}}\right)\left(g\left(x_{i}^{\mathrm{o}}, 0\right)-\bar{g}^{\mathrm{o}}\right)^{\prime}$. In this case, the sample counterpart of the difference $K(\lambda)-K^{\text {et }}(\lambda)$ is of order $O_{p}\left(\max \left\{n^{-1 / 2}|\lambda|,|\lambda|^{2}\right\}\right)$. Also we can see that the same comment applies to the function $K^{\mathrm{cu}}(\lambda)=-\lambda^{\prime} \bar{g}^{\mathrm{o}}-\frac{1}{2} \lambda^{\prime} \hat{V}^{\mathrm{o}} \lambda$ for the continuous updating GMM, i.e., the sample counterpart of the difference $K(\lambda)-K^{\mathrm{cu}}(\lambda)$ is of order $O_{p}\left(\max \left\{n^{-1 / 2}|\lambda|,|\lambda|^{2}\right\}\right)$. Note that $K^{\mathrm{cu}}(\lambda)$ evaluated at $\lambda=\left(\hat{V}^{\mathrm{o}}\right)^{-1} \bar{g}^{\mathrm{o}}$ is the conventional 
Wald statistic. ${ }^{3}$

The following theorem shows that $K^{\text {tet }}(\lambda)$ can provide an accurate approximation to the tail area probabilities of $T_{n}$.

Theorem 3. Suppose that Assumptions 1 and 2 hold true. Then under $H_{0}: \theta_{0}=0$, it holds

$$
1-F_{p}(n \xi(K(\lambda)))=\left\{1-F_{p}\left(n K^{\mathrm{tet}}(\lambda)\right)\right\}\left(1+r_{n}(\lambda)\right)\left(1+O\left(n^{-1}\right)\right)
$$

for each realization $\left\{x_{i}^{o}\right\}_{i=1}^{n}$, where $r_{n}(\lambda)$ is a nonrandom function of $\lambda$ defined in (19) and its sample counterpart, obtained by replacing $\left\{x_{i}^{\mathrm{o}}\right\}_{i=1}^{n}$ with $\left\{X_{i}\right\}_{i=1}^{n}$, is of order $O_{p}\left(n^{1 / 2}|\lambda|^{2}\right)$.

Theorem 3 shows that the TET statistic can provide an accurate approximation to the tail area probability formula $1-F_{p}(n \xi(K(\lambda)))$ for the ideal statistic $T_{n}$ in (7). The error of this approximation is relative and of (stochastic) order $n^{1 / 2}|\lambda|^{2}$. Therefore, in the normal region for $\lambda=O\left(n^{-1 / 2}\right)$, the relative error is of order $n^{-1 / 2}$. Beyond the normal region, e.g., $\lambda=O\left(n^{-1 / 3}\right)$, the relative error approximation is of order $n^{-1 / 6}$. On the other hand, it is clear from (9) that the function $K^{\mathrm{et}}(\lambda)$ for exponential tilting does not have such a relative error property.

Combining Theorems 2 and 3 (evaluated at $\lambda=\hat{\lambda}^{\circ}$ ), we can establish the equality in (4). Therefore, as far as the $p$-value $p^{\text {tet }}$ for the TET statistic is well approximated by the one $p^{\text {ideal }}$ for the ideal statistic $T_{n}$, we can argue that the asymptotic approximation for the TET tends to be accurate particularly in the tail area as illustrated in Figure 1 for the benchmark simulation.

\section{General case}

In this section, we generalize the theoretical results obtained in the last section for testing composite hypotheses (Section 3.1) and overidentifying restrictions (Section 3.2).

\subsection{Composite hypothesis test}

In this subsection, we extend the results for the benchmark case to composite hypothesis testing. We first consider just-identified moment conditions, and then discuss how to adapt our results for overidentified models.

\footnotetext{
${ }^{3}$ Similarly, even if we consider the class of generalized empirical likelihood criterion by Newey and Smith (2004) (i.e., $K^{\text {gel }}(\lambda)=\frac{1}{n} \sum_{i=1}^{n} \rho\left(\lambda^{\prime} g\left(x_{i}^{\text {o }}, 0\right)\right)$ for a concave function $\rho$ ), we can see that the sample counterpart of the difference $K(\lambda)-K^{\text {gel }}(\lambda)$ is of order $O_{p}\left(\max \left\{n^{-1 / 2}|\lambda|,|\lambda|^{2}\right\}\right)$. However, we conjecture that a 'tilted' version of the generalized empirical likelihood $K^{\text {tgel }}(\lambda)=\sum_{i=1}^{n} \hat{\pi}_{\rho, i}^{\mathrm{o}} \rho\left(\lambda^{\prime} g\left(x_{i}^{\mathrm{o}}, 0\right)\right)$ will exhibit a similar property as $K^{\text {tet }}(\lambda)$ in Theorem 3, where $\hat{\pi}_{\rho, i}^{\mathrm{o}}=\rho_{1}\left(\hat{\lambda}_{\rho}^{\mathrm{o}} g\left(x_{i}^{\mathrm{o}}, 0\right)\right) / \sum_{i=1}^{n} \rho_{1}\left(\hat{\lambda}_{\rho}^{\mathrm{o} \prime} g\left(x_{i}^{\mathrm{o}}, 0\right)\right)$ is the generalized empirical likelihood implied probability with $\rho_{1}(v)=\partial \rho(v) / \partial v$ and $\hat{\lambda}_{\rho}^{\mathrm{o}}=\arg \max _{\lambda} \sum_{i=1}^{n} \rho\left(\lambda^{\prime} g\left(x_{i}^{\mathrm{o}}, 0\right)\right)$. We note that in contrast to the (observed) TET statistic $t_{n}^{\text {tet }}=2 K^{\text {tet }}\left(\hat{\lambda}^{\circ}\right)$, its generalized empirical likelihood counterpart $t_{n}^{\text {gel }}=2 K^{\text {tgel }}\left(\hat{\lambda}^{o}\right)$ involves two Lagrange multipliers, $\hat{\lambda}_{\rho}^{o}$ and $\hat{\lambda}^{\circ}$. Full investigation of such an extension is left for future research.
} 


\subsubsection{Just-identified case}

Consider just-identified moment conditions $\mathbb{E}\left[g\left(X, \theta_{10}, \theta_{20}\right)\right]=0$, where $\theta_{10}$ and $\theta_{20}$ are $p_{1^{-}}$and $p_{2}$-dimensional parameters, respectively, and $g$ is a $p=p_{1}+p_{2}$ dimensional vector of moment functions. Suppose we wish to test the null hypothesis $H_{0}: \theta_{20}=0$ against the two-sided alternative $H_{1}: \theta_{20} \neq 0$. In this case, the conventional exponential tilting statistic may be written as

$$
T_{n, c}^{\mathrm{et}}=-2 \max _{\theta_{1} \in \Theta_{1}} \log \left(\frac{1}{n} \sum_{i=1}^{n} e^{\hat{\lambda}\left(\theta_{1}\right)^{\prime} g\left(X_{i}, \theta_{1}, 0\right)}\right),
$$

where $\hat{\lambda}\left(\theta_{1}\right)$ solves $\sum_{i=1}^{n} e^{\hat{\lambda}\left(\theta_{1}\right)^{\prime} g\left(X_{i}, \theta_{1}, 0\right)} g\left(X_{i}, \theta_{1}, 0\right)=0$ for each $\theta_{1}$. It is known that $n T_{n, c}^{\text {et }}$ converges in distribution to the $\chi_{p_{2}}^{2}$ distribution under $H_{0}: \theta_{20}=0$. Let $\tilde{\theta}_{1}$ be a solution of the above constrained maximization for $\theta_{1}$ and $\tilde{\theta}=\left(\tilde{\theta}_{1}^{\prime}, 0^{\prime}\right)^{\prime}$. The TET statistic for the composite hypothesis is constructed as

$$
T_{n, c}^{\mathrm{tet}}=2 \log \left(\sum_{i=1}^{n} \tilde{\pi}_{i} e^{\tilde{\lambda}^{\prime} g\left(X_{i}, \tilde{\theta}\right)}\right)=2\left[\log \left(\sum_{i=1}^{n} e^{2 \tilde{\lambda}^{\prime} g\left(X_{i}, \tilde{\theta}\right)}\right)-\log \left(\sum_{i=1}^{n} e^{\tilde{\lambda}^{\prime} g\left(X_{i}, \tilde{\theta}\right)}\right)\right]
$$

where $\tilde{\pi}_{i}=\frac{e^{\tilde{\lambda}^{\prime} g\left(X_{i}, \tilde{\theta}\right)}}{\sum_{j=1}^{n} e^{\tilde{\nu}^{\prime} g\left(X_{j}, \tilde{\theta}\right)}}$ and $\tilde{\lambda}$ solves $\sum_{i=1}^{n} e^{\tilde{\lambda}^{\prime} g\left(X_{i}, \tilde{\theta}\right)} g\left(X_{i}, \tilde{\theta}\right)=0$. Similar to the last section, we consider the ideal but infeasible statistic

$$
T_{n, c}=2 K\left(\tilde{\lambda}, \tilde{\theta}_{1}(\tilde{\lambda})\right)
$$

where $K\left(\lambda, \theta_{1}\right)=\log \mathbb{E}\left[e^{\lambda^{\prime} g\left(X, \theta_{1}, 0\right)}\right]$ and $\tilde{\theta}_{1}(\lambda)$ solves $\mathbb{E}\left[e^{\lambda^{\prime} g\left(X, \tilde{\theta}_{1}(\lambda), 0\right)} \lambda^{\prime}\left(\frac{\partial g\left(X, \tilde{\theta}_{1}(\lambda), 0\right)}{\partial \theta_{1}^{\prime}}\right)\right]=0$ for each $\lambda$. To analyze the relation between the $T_{n, c}^{\text {tet }}$ and $T_{n, c}$ consider the function $K^{\text {tet }}\left(\lambda, \theta_{1}\right)=$ $\log \left(\sum_{i=1}^{n} \tilde{\pi}_{i}^{\mathrm{o}} e^{\lambda^{\prime} g\left(x_{i}^{\mathrm{o}}, \theta_{1}, 0\right)}\right)$, where $\left\{\tilde{\pi}_{i}^{\mathrm{o}}\right\}_{i=1}^{n}$ are the observed values of $\left\{\tilde{\pi}_{i}\right\}_{i=1}^{n}$. Note that the observed value of $T_{n, c}^{\text {tet }}$ is given by $t_{n, c}^{\text {tet }}=2 K^{\text {tet }}\left(\tilde{\lambda}^{\mathrm{o}}, \tilde{\theta}_{1}^{\text {tet }}\left(\tilde{\lambda}^{\mathrm{o}}\right)\right)$, where $\tilde{\lambda}^{\mathrm{o}}$ is the observed value of $\tilde{\lambda}$ and $\tilde{\theta}_{1}^{\text {tet }}(\lambda)$ solves $\frac{1}{n} \sum_{i=1}^{n} e^{\lambda^{\prime} g\left(x_{i}^{\circ}, \tilde{\theta}_{1}^{\text {tet }}(\lambda), 0\right)} \lambda^{\prime}\left(\frac{\partial g\left(x_{i}^{\mathrm{o}}, \tilde{\theta}_{1}^{\text {tet }}(\lambda), 0\right)}{\partial \theta_{1}^{\prime}}\right)=0$ for each $\lambda$. To analyze the properties of the TET statistic $T_{n, c}^{\text {tet }}$, we modify Assumption 1 as follows.

Assumption 1'. $\left\{X_{i}\right\}_{i=1}^{n}$ is i.i.d., $\theta_{10} \in \operatorname{int} \Theta_{1}$ is the unique solution of $\mathbb{E}\left[g\left(X, \theta_{10}, \theta_{20}\right)\right]=0$, $\Theta_{1}$ is compact, $g\left(x, \theta_{1}, \theta_{20}\right)$ is continuous at each $\theta_{1} \in \Theta_{1}$ and is continuously differentiable in a neighborhood $\mathcal{N}$ of $\theta_{10}$ for almost every $x, \mathbb{E}\left[\sup _{\theta_{1} \in \Theta_{1}}\left|g\left(X, \theta_{1}, \theta_{20}\right)\right|^{\zeta}\right]<\infty$ for some $\zeta>2, \mathbb{E}\left[\sup _{\theta_{1} \in \mathcal{N}}\left|\partial g\left(X, \theta_{1}, \theta_{20}\right) / \partial \theta_{1}^{\prime}\right|\right]<\infty, \mathbb{E}\left[\partial g\left(X, \theta_{10}, \theta_{20}\right) / \partial \theta_{1}^{\prime}\right]$ is full column rank, and $\mathbb{E}\left[g\left(X, \theta_{0}\right) g\left(X, \theta_{0}\right)^{\prime}\right]$ is nonsingular.

The relative error properties of $T_{n, c}$ and $T_{n, c}^{\text {tet }}$ are presented as follows. 
Theorem 4. Suppose that Assumption 1' holds true and that Assumption 2 is satisfied with $\tilde{g}\left(X, \theta_{0}\right)$ in (21) instead of $g(X, 0)$. Then under $H_{0}: \theta_{20}=0$,

(i) the ideal statistic $T_{n, c}$ satisfies

$$
\operatorname{Pr}\left\{n T_{n, c} \geq n t: F\right\}=\left\{1-F_{p_{2}}\left(n \xi_{c}(t)\right)\right\}\left(1+O\left(n^{-1}\right)\right)
$$

uniformly over $t \in(0, \varepsilon)$ for some $\varepsilon>0$, where $\xi_{c}(t)$ is defined as in $\xi(t)$ (by replacing $g(X, 0)$ with $\left.\tilde{g}\left(X, \theta_{0}\right)\right)$,

(ii) $K^{\text {tet }}\left(\lambda, \theta_{1}\right)$ satisfies

$$
1-F_{p_{2}}\left(n \xi_{c}\left(K\left(\lambda, \tilde{\theta}_{1}(\lambda)\right)\right)\right)=\left\{1-F_{p_{2}}\left(n K^{\mathrm{tet}}\left(\lambda, \tilde{\theta}_{1}^{\mathrm{tet}}(\lambda)\right)\right)\right\}\left(1+r_{n, c}(\lambda)\right)\left(1+O\left(n^{-1}\right)\right),
$$

for each realization $\left\{x_{i}^{\circ}\right\}_{i=1}^{n}$, where $r_{n, c}(\lambda)$ is a nonrandom function of $\lambda$ and its sample counterpart, obtained by replacing $\left\{x_{i}^{\mathrm{o}}\right\}_{i=1}^{n}$ with $\left\{X_{i}\right\}_{i=1}^{n}$, is of order $O_{p}\left(n^{1 / 2}|\lambda|^{2}\right)$.

Theorem 4 (i) highlights the desirable relative error property of the ideal statistic $T_{n, c}$. Theorem 4 (ii) shows that the TET statistic can be expected to provide accurate approximations of the tail area probabilities of the ideal statistic $T_{n, c}$.

We can compare with the saddlepoint statistic introduced in Ma and Ronchetti (2011). In this case, their statistic is written as

$$
2\left[\log \left(\sum_{i=1}^{n} e^{\tilde{\lambda}^{\prime} g\left(X_{i}, \tilde{\theta}\right)}\right)-\log \left(\sum_{i=1}^{n} e^{\tilde{\lambda}^{\prime} g\left(X_{i}, \tilde{\theta}\right)+\hat{\mu}^{\prime} g\left(X_{i}, \bar{\theta}_{1}, \hat{\theta}_{2}\right)}\right)\right],
$$

where $\hat{\theta}=\left(\hat{\theta}_{1}^{\prime}, \hat{\theta}_{2}^{\prime}\right)^{\prime}$ solves $\sum_{i=1}^{n} g\left(X_{i}, \hat{\theta}\right)=0, \hat{\mu}$ and $\bar{\theta}_{1}$ solve $\sum_{i=1}^{n} \tilde{\pi}_{i} e^{\hat{\mu}^{\prime} g\left(X_{i}, \bar{\theta}_{1}, 0\right)} g\left(X_{i}, \bar{\theta}_{1}, \hat{\theta}_{2}\right)=0$ and $\hat{\mu}^{\prime} \sum_{i=1}^{n} \tilde{\pi}_{i} e^{\hat{\mu}^{\prime} g\left(X_{i}, \hat{\theta}\right)} \partial g\left(X_{i}, \bar{\theta}_{1}, \hat{\theta}_{2}\right) / \partial \theta_{1}^{\prime}=0$. Note that this saddlepoint statistic requires to solve several equations to obtain $\hat{\theta}, \tilde{\theta}, \bar{\theta}_{1}, \tilde{\lambda}$, and $\hat{\mu}$. In contrast, the TET statistic $n T_{n, c}^{\text {tet }}$ only requires to solve for $\tilde{\theta}$ and $\tilde{\lambda}^{4}$

\footnotetext{
${ }^{4}$ Although the TET statistic $n T_{n, c}^{\text {tet }}$ takes a simpler form than Ma and Ronchetti's (2011), we still need to compute $\tilde{\theta}=\left(\tilde{\theta}_{1}^{\prime}, 0^{\prime}\right)^{\prime}$ and $\tilde{\lambda}$ to implement our test. The computation of $\tilde{\lambda}$ (for a given $\tilde{\theta}$ ) can be formulated as a convex optimization problem so that Newton iterations can be applied. We use the matlab codes provided by Kirill Evdokimov and Yuichi Kitamura (available at https://www.kirillevdokimov.com/EL_codes.zip) for our numerical studies. On the other hand, the computation of $\tilde{\theta}$ typically requires a nested optimization algorithm, where the inner loop computes $\hat{\lambda}\left(\theta_{1}\right)$ for each $\theta_{1}$, and the outer loop computes $\tilde{\theta}_{1}$ in (10). Similar to $\tilde{\lambda}$, the inner loop is typically a convex optimization problem which can be implemented by Newton iterations. The outer loop is a general nonlinear optimization problem, and we employ a quasi-Newton method, which works well in our numerical studies. See Kitamura (2007) for a detailed discussion on the nested optimization algorithm.
} 


\subsubsection{Overidentified case}

The results in Theorem 4 can be adapted to overidentified models. For overidentified moment conditions $\mathbb{E}\left[g\left(X, \theta_{10}, \theta_{20}\right)\right]=0$, where $\theta_{10}$ and $\theta_{20}$ are $p_{1^{-}}$and $p_{2}$-dimensional, respectively, and $g$ is $d>p_{1}+p_{2}$ dimensional, suppose we wish to test the null hypothesis $H_{0}: \theta_{20}=0$ against the two-sided alternative $H_{1}: \theta_{20} \neq 0$. Indeed this testing problem can be written as the one for $H_{0}: \theta_{20}=0$ in the following augmented moment conditions

$$
\mathbb{E}\left[h\left(X, \mu_{0}, \theta_{10}, \theta_{20}\right)\right]=\mathbb{E}\left[\begin{array}{c}
e^{\mu_{0}^{\prime} g\left(X, \theta_{10}, \theta_{20}\right)} g\left(X, \theta_{10}, \theta_{20}\right) \\
e^{\mu_{0}^{\prime} g\left(X_{i}, \theta_{10}, \theta_{20}\right)}\left(\frac{\partial g\left(X, \theta_{10}, \theta_{20}\right)}{\partial\left(\theta_{1}^{\prime}, \theta_{2}^{\prime}\right)}\right)^{\prime} \mu_{0}
\end{array}\right]=0 .
$$

Note that due to the additional $d$-dimensional parameters $\mu_{0}$, this moment condition model is just-identified. Therefore, the results in Section 3.1.1 for the just-identified case can be adapted by replacing the moment function $g(\cdot)$ with $h(\cdot)$, and nuisance parameters $\theta_{10}$ with $\vartheta_{10}=\left(\theta_{10}^{\prime}, \mu_{0}^{\prime}\right)^{\prime}$. Let $\tilde{\vartheta}_{1}=\arg \max _{\vartheta_{1}} \log \left(\frac{1}{n} \sum_{i=1}^{n} e^{\hat{\lambda}\left(\vartheta_{1}\right)^{\prime} h\left(X_{i}, \vartheta_{1}, 0\right)}\right)$, where $\hat{\lambda}\left(\vartheta_{1}\right)$ solves $\sum_{i=1}^{n} e^{\hat{\lambda}\left(\vartheta_{1}\right)^{\prime} h\left(X_{i}, \vartheta_{1}, 0\right)} h\left(X_{i}, \vartheta_{1}, 0\right)=0$ for each $\vartheta_{1}$. The TET statistic is defined as

$$
T_{n, c 1}^{\mathrm{tet}}=2 \log \left(\sum_{i=1}^{n} \tilde{\pi}_{i} e^{\tilde{\lambda}^{\prime} h\left(X_{i}, \tilde{\vartheta}\right)}\right)
$$

where $\tilde{\vartheta}=\left(\tilde{\vartheta}_{1}^{\prime}, 0^{\prime}\right)^{\prime}, \tilde{\pi}_{i}=\frac{e^{\tilde{\lambda}^{\prime} h\left(X_{i}, \tilde{\vartheta}\right)}}{\sum_{j=1}^{n} e^{\tilde{\lambda}^{\prime} h\left(X_{j}, \tilde{\vartheta}\right)}}$, and $\tilde{\lambda}$ solves $\sum_{i=1}^{n} e^{\tilde{\lambda}^{\prime} h\left(X_{i}, \tilde{\vartheta}\right)} h\left(X_{i}, \tilde{\vartheta}\right)=0$. By applying the results in Theorem 4, analogous relative error properties for $T_{n, c 1}^{\mathrm{tet}}$ can be derived.

\subsection{Overidentifying restriction test}

In this subsection, we consider the case of overidentifying moment restrictions $\mathbb{E}\left[g\left(X, \theta_{0}\right)\right]=$ 0 , where the dimension $d$ of the moment functions $g$ is larger than the dimension $p$ of the unknown parameters $\theta_{0}$. In particular, we focus on testing overidentifying restrictions, i.e., $H_{0}: \mathbb{E}[g(X, \theta)]=0$ for some $\theta$ against $H_{1}: \mathbb{E}[g(X, \theta)] \neq 0$ for any $\theta$. This is a specification testing problem for the model specified by moment restrictions. In this case, the conventional exponential tilting statistic may be written as

$$
T_{n, v}^{\mathrm{et}}=-2 \max _{\theta} \log \left(\frac{1}{n} \sum_{i=1}^{n} e^{\hat{\lambda}(\theta)^{\prime} g\left(X_{i}, \theta\right)}\right)
$$

where $\hat{\lambda}(\theta)$ is defined in the last subsection. Based on Newey and Smith (2004), we can show that $n T_{n, v}^{\mathrm{et}}$ converges in distribution to the $\chi_{d-p}^{2}$ distribution under the null hypothesis. Let $\bar{\theta}$ be the maximizer of the above optimization problem. The TET statistic for the overidentifying 
restriction test is constructed as

$$
T_{n, v}^{\mathrm{tet}}=2 \log \left(\sum_{i=1}^{n} \bar{\pi}_{i} e^{\bar{\lambda}^{\prime} g\left(X_{i}, \bar{\theta}\right)}\right)=2\left[\log \left(\sum_{i=1}^{n} e^{2 \bar{\lambda}^{\prime} g\left(X_{i}, \bar{\theta}\right)}\right)-\log \left(\sum_{i=1}^{n} e^{\bar{\lambda}^{\prime} g\left(X_{i}, \bar{\theta}\right)}\right)\right],
$$

where $\bar{\pi}_{i}=\frac{e^{\bar{\lambda}^{\prime} g\left(X_{i}, \bar{\theta}\right)}}{\sum_{j=1}^{n} e^{\bar{\lambda}^{\prime} g\left(X_{j}, \bar{\theta}\right)}}$ and $\bar{\lambda}$ solves $\sum_{i=1}^{n} e^{\bar{\lambda}^{\prime} g\left(X_{i}, \bar{\theta}\right)} g\left(X_{i}, \bar{\theta}\right)=0$. In this case, the ideal but infeasible statistic is defined as

$$
T_{n, v}=2 K(\bar{\lambda}, \bar{\theta}(\lambda)) .
$$

where $\bar{\theta}(\lambda)$ solves $\mathbb{E}\left[e^{\lambda^{\prime} g(X, \bar{\theta}(\lambda))} \lambda^{\prime}\left(\frac{\partial g(X, \bar{\theta}(\lambda))}{\partial \theta^{\prime}}\right)\right]=0$. Next, consider the function $K^{\text {tet }}(\lambda, \theta)=$ $\log \left(\sum_{i=1}^{n} \bar{\pi}_{i}^{\mathrm{o}} e^{\lambda^{\prime} g\left(x_{i}^{\mathrm{o}}, \theta\right)}\right)$, where $\left\{\bar{\pi}_{i}^{\mathrm{o}}\right\}_{i=1}^{n}$ are the observed values of $\left\{\bar{\pi}_{i}\right\}_{i=1}^{n}$. Note that the observed value of $T_{n, v}^{\text {tet }}$ is given by $t_{n, v}^{\text {tet }}=2 K^{\text {tet }}\left(\bar{\lambda}^{\mathrm{o}}, \bar{\theta}^{\text {tet }}\left(\bar{\lambda}^{\mathrm{o}}\right)\right)$, where $\bar{\lambda}^{\mathrm{o}}$ is the observed value of $\bar{\lambda}$ and $\bar{\theta}^{\text {tet }}(\lambda)$ solves $\frac{1}{n} \sum_{i=1}^{n} e^{\lambda^{\prime} g\left(x_{i}^{\mathrm{o}}, \bar{\theta}^{\text {tet }}(\lambda)\right)} \lambda^{\prime}\left(\frac{\partial g\left(x_{i}^{\mathrm{o}}, \bar{\theta}^{\mathrm{tet}}(\lambda)\right)}{\partial \theta^{\prime}}\right)=0$ for each $\lambda$. To analyze the properties of the TET statistic $T_{n, v}^{\text {tet }}$, we modify Assumption 1 as follows.

Assumption 1". $\left\{X_{i}\right\}_{i=1}^{n}$ is i.i.d., $\theta_{0} \in \operatorname{int} \Theta$ is the unique solution of $\mathbb{E}\left[g\left(X, \theta_{0}\right)\right]=0, \Theta$ is compact, $g(x, \theta)$ is continuous at each $\theta \in \Theta$ and is continuously differentiable in a neighborhood $\mathcal{N}$ of $\theta_{0}$ for almost every $x, \mathbb{E}\left[\sup _{\theta \in \Theta}|g(X, \theta)|^{\zeta}\right]<\infty$ for some $\zeta>2, \mathbb{E}\left[\sup _{\theta \in \mathcal{N}}\left|\partial g(X, \theta) / \partial \theta^{\prime}\right|\right]<$ $\infty, \mathbb{E}\left[\partial g\left(X, \theta_{0}\right) / \partial \theta^{\prime}\right]$ is full column rank, and $\mathbb{E}\left[g\left(X, \theta_{0}\right) g\left(X, \theta_{0}\right)^{\prime}\right]$ is nonsingular.

The relative error properties of $T_{n, v}$ and $T_{n, v}^{\text {tet }}$ are presented as follows.

Theorem 5. Suppose that Assumption 1" holds true and the adapted version of Assumption 2 is satisfied. Then under $H_{0}: \mathbb{E}\left[g\left(X, \theta_{0}\right)\right]=0$,

(i) the ideal statistic $T_{n, v}$ satisfies

$$
\operatorname{Pr}\left\{n T_{n, v} \geq n t: F\right\}=\left\{1-F_{d-p}\left(n \xi_{v}(t)\right)\right\}\left(1+O\left(n^{-1}\right)\right)
$$

uniformly over $t \in(0, \varepsilon)$ for some $\varepsilon>0$, where $\xi_{v}(t)$ is defined as in $\xi(t)$,

(ii) $K^{\text {tet }}(\lambda, \theta)$ satisfies

$$
1-F_{d-p}\left(n \xi_{v}(K(\lambda, \bar{\theta}(\lambda)))\right)=\left\{1-F_{d-p}\left(n K^{\mathrm{tet}}\left(\lambda, \bar{\theta}^{\mathrm{tet}}(\lambda)\right)\right)\right\}\left(1+r_{n, v}(\lambda)\right)\left(1+O\left(n^{-1}\right)\right),
$$

for each realization $\left\{x_{i}^{\circ}\right\}_{i=1}^{n}$, where $r_{n, v}(\lambda)$ is a nonrandom function of $\lambda$ and its sample counterpart, obtained by replacing $\left\{x_{i}^{\mathrm{o}}\right\}_{i=1}^{n}$ with $\left\{X_{i}\right\}_{i=1}^{n}$, is of order $O_{p}\left(n^{1 / 2}|\lambda|^{2}\right)$.

Theorem 5 shows that the desirable relative error properties of the TET statistic continue to hold in overidentified moment condition models. 


\section{Simulation for general case}

In this section, we evaluate the finite sample performance of the TET statistic for the general case considered in the last section. We generate random samples $\left\{W_{i}\right\}_{i=1}^{n}=\left\{Y_{i}, X_{i}, Z_{i}^{\prime}\right\}_{i=1}^{n}$ of size $n=40$ according to

$$
\begin{aligned}
Y_{i} & =\theta_{1}+\theta_{2} X_{i}+U_{i}, \\
X_{i} & =Z_{i}^{\prime} \pi+V_{i},
\end{aligned}
$$

where $\pi=(c, c)^{\prime}$ and $Z_{i}=\left(1, Z_{2 i}\right)^{\prime}$ with $Z_{2 i} \sim N(0,1)$. For each Monte Carlo replication, we set the value of $c$ to fix the value of the concentration parameter $\delta^{2}=\pi^{\prime}\left(\sum_{i=1}^{n} Z_{i} Z_{i}^{\prime}\right) \pi$ (given the realized values of $\left.Z_{i}\right)$. We set $\left(\theta_{1}, \theta_{2}\right)=(0,0)$ for the true parameter vector. The error terms are generated as $\left(U_{i}, V_{i}\right)=\left(\epsilon_{1 i}, 0.8 \epsilon_{1 i}+\sqrt{1-0.8^{2}} \epsilon_{2 i}\right)$, where $\epsilon_{1 i}$ and $\epsilon_{2 i}$ are independent. The number of Monte Carlo replications is 10,000 for all cases. All figures reporting the simulation results are presented in Appendix B.

\subsection{Composite hypothesis testing for $H_{0}: \theta_{2}=0$}

First, as an illustration for the composite hypothesis testing in Section 3.1, we consider testing the null hypothesis $H_{0}: \theta_{2}=0$. For the model in (14), we consider three cases: (i) $\epsilon_{1 i}, \epsilon_{2 i} \sim N(0,1)$ and $\delta^{2}=50$, (ii) $\epsilon_{1 i}, \epsilon_{2 i} \sim N(0,1)$ and $\delta^{2}=10$, and (iii) $\epsilon_{1 i}, \epsilon_{2 i} \sim\left(\chi_{3}^{2}-3\right) / \sqrt{6}$ (standardized $\chi_{3}^{2}$ ) and $\delta^{2}=50$. Case (i) is a baseline setup, (ii) is for investigating the effect of weaker instruments, and (iii) is for the effect of asymmetric distributions of the error terms. The cases of even weaker instruments with a weakly identified nuisance parameter are investigated in Section 4.3.

We compare the proposed TET statistic $T_{n, c}^{\mathrm{et}}$ in (11) with the Wald statistic based on the GMM estimator and exponential tilting (ET) statistic $T_{n, c}^{\mathrm{et}}$ in (10). All the statistics converge in distribution to the $\chi_{1}^{2}$ distribution under $H_{0}$. Figure 2 reports the q-q plots of the empirical quantiles of these test statistics against those of the $\chi_{1}^{2}$ distribution for the cases (i)-(iii).

Overall, the empirical quantiles of the TET statistic are very close to those of the limiting distribution. These q-q plots of the TET statistics are not sensitive to weaker instruments (as shown in Figure 2(b)) or asymmetric error distributions (as shown in Figure 2(c)). On the other hand, the quantiles of the conventional Wald and ET statistics tend to be larger than the ones of the limiting distribution. In particular, the Wald statistic is worse for all the cases. Such patterns of the q-q plots for the Wald and ET statistics are commonly observed in the literature 
(e.g., Imbens, Spady and Johnson, 1998). ${ }^{5}$ Interestingly, the weaker instruments induce more severe size distortions for the Wald test (see Figure 2(b)).

We also investigate the power properties of the tests for $H_{0}: \theta_{2}=0$ under the alternative hypotheses $H_{1}: \theta_{2}=0+\Delta$ for different values of $\Delta$. Figures 3 displays the calibrated power curves of all the tests at $5 \%$ significance level (i.e., the rejection frequencies of these tests, where the critical values are given by the Monte Carlo 95th percentiles of these test statistics under $H_{0}$ ) for the cases (i)-(iii). The results suggest that the proposed TET tests exhibit good calibrated power. Indeed the calibrated power curves of the TET are very close to those of the ET, and do not show declining power for some negative values of $\Delta$ as in the Wald test.

\section{$4.2 \quad$ Specification testing}

Second, as an illustration for specification testing in Section 3.2, we consider testing the overidentifying restrictions $H_{0}: \mathbb{E}[Z(Y-\theta X)]=0$ for some $\theta$ against $H_{1}: \mathbb{E}[Z(Y-\theta X)] \neq 0$ for any $\theta$. We compare the proposed TET statistic $T_{n, v}^{\text {tet }}$ in (13) with the J-statistic based on GMM, ${ }^{6}$ and ET statistic $T_{n, v}^{\mathrm{et}}$ in (12). All the statistics converge in distribution to the $\chi_{1}^{2}$ distribution under $H_{0}$.

In addition to the cases (i) and (ii) above, we consider the case of heteroskedastic error terms (iv) $Y_{i}=\theta_{1}+\theta_{2} X_{i}+\left|Z_{i}\right| U_{i}, \epsilon_{1 i}, \epsilon_{2 i} \sim N(0,1)$, and $\delta^{2}=50$. Figure 4 reports the q-q plots of the empirical quantiles of these test statistics against those of the $\chi_{1}^{2}$ distribution for the normal disturbance case.

Although the patterns of the plots are different, we obtain similar conclusions as the simulations for composite hypothesis testing. Overall, the empirical quantiles of the TET statistic are very close to those of the limiting distribution compared to the ET and J statistics. The performances of the ET and $\mathrm{J}$ statistics are comparable. It is interesting to note that the $\mathrm{q}-\mathrm{q}$ plots of the TET statistic are not sensitive to heteroskedastic error terms. On the other hand, we can see that heteroskedastic errors deteriorate the size properties of the ET and J statistics.

Overall, our simulation results are encouraging: the TET statistic performs excellently compared to the existing statistics.

\footnotetext{
${ }^{5}$ In the working paper version (available at: http://sticerd.lse.ac.uk/dps/em/em593.pdf), we also report the results for the case of $n=80$ and the empirical cumulative distribution functions (ECDFs) of the $p$-values for all the tests considered in Figures 2 and 4 below. The accuracy of the ET statistic improves as the sample size increases. However, the TET always outperforms the ET and Wald. Furthermore, based on the ECDFs for the $p$-values, we can see the degrees of size distortions of these statistics. As consistent with the q-q plots, the asymptotic test by the TET shows better size properties (particularly for the region of nominal sizes less than $0.10)$ than other asymptotic tests.

${ }^{6}$ Letting $g\left(W_{i}, \theta\right)=Z_{i}\left(Y_{i}-\theta X_{i}\right)$ and $\bar{g}(\theta)=n^{-1} \sum_{i=1}^{n} g\left(W_{i}, \theta\right)$, the version of the J-test statistic considered here is $\left.J=n \min _{\theta} \bar{g}(\theta)^{\prime}\left[n^{-1} \sum_{i=1}^{n} g\left(W_{i}, \breve{\theta}\right) g W_{i}, \check{\theta}\right)\right]^{-1} \bar{g}(\theta)$, where $\check{\theta}$ is the two stage least square estimator.
} 


\subsection{Additional results: Weak instruments}

Finally, we consider the situation where the instrumental variable $Z$ is very weak (i.e., $\delta^{2}=5$ and 1). There is no guarantee that the proposed TET statistic works well under the partial or weak identification; see, Phillips (1989), Staiger and Stock (1997), or Phillips (1980) for an exact analysis with Gaussian errors. In particular, the full rank conditions for the Jacobians of $g$ (with respect to partially or weakly identified parameters) in Assumptions 1' and 1" typically fail, and our asymptotic approximations will be invalid. Although its theoretical analysis is left for future research, we here illustrate finite sample performances of the TET statistic under weak instruments.

Specifically, we consider three cases: (i') $\epsilon_{1 i}, \epsilon_{2 i} \sim N(0,1)$ and $\delta^{2}=5$, (ii') $\epsilon_{1 i}, \epsilon_{2 i} \sim N(0,1)$ and $\delta^{2}=1$, and (iii') $\epsilon_{1 i}, \epsilon_{2 i} \sim\left(\chi_{3}^{2}-3\right) / \sqrt{6}$ (standardized $\left.\chi_{3}^{2}\right)$ and $\delta^{2}=5$. Figures 5 and 6 report the q-q plots of the empirical quantiles of the test statistics against those of the $\chi_{1}^{2}$ distribution for testing $H_{0}: \theta_{2}=0$ and $H_{0}: \theta_{1}=0$, respectively. We note that for testing $H_{0}: \theta_{1}=0$, the nuisance parameter $\theta_{2}$ is weakly identified and we conjecture that the TET test will be asymptotically invalid based on the existing literature of weak instruments (e.g., Andrews and Stock, 2007, for a survey).

For testing $H_{0}: \theta_{2}=0$, where the nuisance parameter $\theta_{1}$ is strongly identified, the q-q plots in Figure 5 are similar to the ones in Figure 2, and the TET statistics are not sensitive to very weak instruments. However, for testing $H_{0}: \theta_{1}=0$, where the nuisance parameter $\theta_{2}$ is weakly identified, the q-q plots in Figure 6 suggest that the quantiles of the TET statistics deviate from the ones of the $\chi^{2}$ limiting distribution. ${ }^{7}$ In particular, the TET is worse than other statistics for the case (ii'). It is an interesting direction of future research to see whether the TET statistic can be modified to be robust to weak instruments or identification.

\section{Conclusion}

In this paper, we modify the exponential tilting statistic by introducing additional tilting weights to estimate cumulant functions, and propose a novel statistic, called the tilted exponential tilting (TET) statistic. Simulation studies show that the asymptotic $p$-values of the TET statistic are highly accurate in the tails, and we provide a theoretical explanation for this accuracy by analyzing its relative error properties for the approximation of tail area probabilities. In particular, the proposed $p$-values accurately approximate those of an infeasible saddlepoint statistic, which

\footnotetext{
${ }^{7}$ In the preliminary simulation study, we considered overidentification testing under the cases (i')-(iii'). The q-q plots also indicate that the TET statistic for overidentifying restrictions is not robust to very weak instruments.
} 
has relative errors of order $n^{-1}$ both in normal and large deviation regions. Our TET test allows both just- and over-identified moment condition models, and can be applied for both (possibly composite) parameter hypotheses and overidentifying restriction testing problems.

There are at least two important directions for future research. First, as repeatedly mentioned, the present analysis lacks theoretical evaluations of closeness of the $p$-values for the infeasible statistic to the ones for the proposed TET statistic. Although it is a substantial challenge given the existing results of saddlepoint approximations, this theoretical gap should be addressed. Another interesting direction is to investigate theoretical properties of the TET statistic under weak and/or many moment conditions, and to develop a modified statistic that is not only accurate in the tails but also robust to weak and/or many moment conditions. 


\section{A Proofs}

\section{A.1 Proof of Theorem 1}

Let $g_{i}=g\left(X_{i}, 0\right)$. Using $\sum_{i=1}^{n} \hat{\pi}_{i}=1$ and $\sum_{i=1}^{n} \hat{\pi}_{i} g_{i}=0$, an expansion around $\hat{\lambda}=0$ implies

$$
n T_{n}^{\mathrm{tet}}=n \hat{\lambda}^{\prime}\left[\sum_{i=1}^{n} \hat{\pi}_{i} e^{\bar{\lambda}^{\prime} g_{i}} g_{i} g_{i}^{\prime}\right] \hat{\lambda}
$$

where $\bar{\lambda}$ is a point on the line joining $\hat{\lambda}$ and 0 . Let $\hat{M}=-\frac{1}{n} \sum_{j=1}^{n} e^{\hat{\lambda}^{\prime} g_{j}}$. An expansion around $\hat{M}=-1$ implies

$$
\begin{aligned}
n T_{n}^{\mathrm{tet}} & =-\hat{M}^{-1} n \hat{\lambda}^{\prime}\left[\frac{1}{n} \sum_{i=1}^{n} e^{(\hat{\lambda}+\bar{\lambda})^{\prime} g_{i}} g_{i} g_{i}^{\prime}\right] \hat{\lambda} \\
& =n \hat{\lambda}^{\prime}\left[\frac{1}{n} \sum_{i=1}^{n} e^{(\hat{\lambda}+\bar{\lambda})^{\prime} g_{i}} g_{i} g_{i}^{\prime}\right] \hat{\lambda}+\bar{M}^{-2} n \hat{\lambda}^{\prime}\left[\frac{1}{n} \sum_{i=1}^{n} e^{(\hat{\lambda}+\bar{\lambda})^{\prime} g_{i}} g_{i} g_{i}^{\prime}\right] \hat{\lambda}(\hat{M}+1) \\
& =T_{1}+T_{2},
\end{aligned}
$$

where $\bar{M}$ is a point on the line joining $\hat{M}$ and -1 . By applying the argument in Newey and Smith (2004, pp. 239-240), we can show $\max _{1 \leq i \leq n}\left|-e^{\hat{\lambda}^{\prime} g_{i}}+1\right| \stackrel{p}{\rightarrow} 0$ and $\max _{1 \leq i \leq n}\left|e^{\bar{\lambda}^{\prime} g_{i}}+1\right| \stackrel{p}{\rightarrow} 0$. An expansion of $\sum_{i=1}^{n} e^{\hat{\lambda}^{\prime} g_{i}} g_{i}=0$ around $\hat{\lambda}=0$ implies

$$
\hat{\lambda}=-\left(\frac{1}{n} \sum_{i=1}^{n} g_{i} g_{i}^{\prime}\right)^{-1}\left(\frac{1}{n} \sum_{i=1}^{n} g_{i}\right)+o_{p}\left(n^{-1 / 2}\right) .
$$

Combining these results,

$$
T_{1}=\left(\frac{1}{\sqrt{n}} \sum_{i=1}^{n} g_{i}\right)^{\prime}\left(\frac{1}{n} \sum_{i=1}^{n} g_{i} g_{i}^{\prime}\right)^{-1}\left(\frac{1}{\sqrt{n}} \sum_{i=1}^{n} g_{i}\right)+o_{p}(1) \stackrel{d}{\rightarrow} \chi_{p}^{2} .
$$

Finally, by $\max _{1 \leq i \leq n}\left|-e^{\hat{\lambda}^{\prime} g_{i}}+1\right| \stackrel{p}{\rightarrow} 0$, it holds $\hat{M}+1 \stackrel{p}{\rightarrow} 0$ and then $T_{2} \stackrel{p}{\rightarrow} 0$. Therefore, the conclusion follows.

\section{A.2 Proof of Theorem 2}

The basic idea of the proof is similar to that of Robinson, Ronchetti and Young (2003, Theorem 1). Let $m(\lambda)=\log \mathbb{E}\left[e^{\lambda^{\prime} \check{g}(X)}\right]$ so that $T_{n}=2 m(\check{\lambda})$. By Assumption 2 , the tail probability of $T_{n}$ 
is approximated as

$$
\begin{aligned}
& \operatorname{Pr}\left\{n T_{n} \geq n t: F\right\}=\operatorname{Pr}\{2 m(\check{\lambda}) \geq t: F\} \\
= & \int_{\{y: 2 m(y) \geq t\}}\left(\frac{n}{2 \pi}\right)^{p / 2} e^{-n h(y)} \frac{\operatorname{det} B(y)}{\sqrt{\operatorname{det} \Sigma(y)}} d y\left(1+O\left(n^{-1}\right)\right) \\
\equiv & A\left(1+O\left(n^{-1}\right)\right) .
\end{aligned}
$$

To evaluate the integral $A$, consider the polar transformation $y \mapsto(r, s)$ (with radius $r$ and angle $s$ ) and another transformation $(r, s) \mapsto(u, s)$ with $u=\sqrt{2 m(y)}$. The Jacobians of these transformations are $J_{1}(y)=\left(y^{\prime} y\right)^{\frac{p-1}{2}}$ and $J_{2}(y)=\frac{\sqrt{y^{\prime} y} \sqrt{2 m(y)}}{m_{1}(y)^{\prime} y}$, respectively, where $m_{1}(y)=$ $d m(y) / d y$. Define the transform $y \mapsto(u, s)$ as $y=\varphi(u, s)$. By the change of variables, the above integral is written as

$$
A=\int_{\sqrt{t}}^{\infty} c_{n} u^{p-1} e^{-n u^{2} / 2}\left\{\int_{S} \delta(u, s) d s\right\} d u
$$

where $c_{n}=n^{p / 2} /\left(2^{p / 2-1} \Gamma(p / 2)\right), S$ is the $p$-dimensional unit sphere, and

$$
\delta(u, s)=\frac{e^{n u^{2} / 2-n h(\varphi(u, s))} \Gamma(p / 2)}{2 \pi^{p / 2} u^{p-1}} \frac{\operatorname{det} B(\varphi(u, s))}{\sqrt{\operatorname{det} \Sigma(\varphi(u, s))}} J_{1}(\varphi(u, s)) J_{2}(\varphi(u, s)) .
$$

We expand each term in $\delta(u, s)$. First, note that

$$
\begin{aligned}
\operatorname{det} B(\varphi(u, s)) & =\operatorname{det} B(0)\left\{1+r \xi_{1}(s)+r^{2} R_{1}(r, s)\right\} \\
\frac{1}{\sqrt{\operatorname{det} \Sigma(\varphi(u, s))}} & =\frac{1}{\sqrt{\operatorname{det} \Sigma(0)}}\left\{1+r \xi_{2}(s)+r^{2} R_{2}(r, s)\right\}
\end{aligned}
$$

where $\xi_{1}$ and $\xi_{2}$ are linear combinations of components of $s$, and $R_{1}$ and $R_{2}$ are uniformly bounded for $r$ bounded. Due to the normalization $\mathbb{E}\left[\check{g}(X) \check{g}(X)^{\prime}\right]=I$, we have $\frac{\operatorname{det} B(0)}{\sqrt{\operatorname{det} \Sigma(0)}}=1$. Thus, other terms are expanded as

$$
\begin{aligned}
e^{n u^{2} / 2-n h(\varphi(u, s))} & =1+r^{2} R_{3}(r, s), \\
J_{1}(y) & =r^{p-1}, \\
J_{2}(y) & =1+r \xi_{4}(s)+r^{2} R_{4}(r, s), \\
u & =r\left\{1+r \xi_{5}(s)+r^{2} R_{5}(r, s)\right\},
\end{aligned}
$$

where $\xi_{4}$ and $\xi_{5}$ are linear combinations of terms of the form $s_{i} s_{j} s_{k}$, and $R_{3}, R_{4}$ and $R_{5}$ are uniformly bounded for $r$ bounded. Combining all these expansions,

$$
\delta(u, s)=\frac{\Gamma(p / 2)}{2 \pi^{p / 2}}\left\{1+u b(s)+u^{2} R_{6}(u, s)\right\}
$$


where $R_{6}$ is uniformly bounded for $r$ bounded, and $b(s)$ is a linear combination of odd functions satisfying $\int_{S} b(s) d s=0$. Integrating over the sphere gives

$$
G(u) \equiv \int_{S} \delta(u, s) d s=1+u^{2} k(u)
$$

for some $k(u)$ bounded over $u \in(0, \varepsilon)$. Also we can see that $d G(u) / d u=u k_{1}(u)$ for some $k_{1}(u)$ bounded over $u \in(0, \varepsilon)$.

From (15)-(17),

$$
\begin{aligned}
\operatorname{Pr}\left\{n T_{n} \geq n t: F\right\} & =\int_{\sqrt{t}}^{\infty} c_{n} u^{p-1} e^{-n u^{2} / 2} G(u) d u\left(1+O\left(n^{-1}\right)\right) \\
& =\int_{\sqrt{t}}^{\infty} c_{n} u^{p-1} e^{-n(u-\log G(u) /(n u))^{2} / 2} d u\left(1+O\left(n^{-1}\right)\right),
\end{aligned}
$$

where the second equality follows from boundedness of $k(u)$ and $k_{1}(u)$. The conclusion follows by the change of variables $v=u-\log G(u) /(n u)$ and boundedness of $k(u)$ and $k_{1}(u)$.

\section{A.3 Proof of Theorem 3}

Using (15)-(17) in the proof of Theorem 2 and integration by parts, we have

$$
1-F_{p}(n \xi(K(\lambda)))=\left\{1-F_{p}(n K(\lambda))\right\}\left(1+O\left(n^{-1}\right)\right)+\frac{c_{n}}{n} K(\lambda)^{\frac{p}{2}} e^{-\frac{n K(\lambda)}{2}}\left[\frac{G(\sqrt{K(\lambda)})-1}{K(\lambda)}\right]
$$

For the first term on the right hand side of (18), the mean-value theorem yields

$$
1-F_{p}(n K(\lambda))=\left\{1-F_{p}\left(n K^{\mathrm{tet}}(\lambda)\right)\right\}\left(1+r_{1 n}(\lambda)\right),
$$

where $r_{1 n}(\lambda)=\frac{e^{-\bar{u}_{\lambda} / 2} \bar{u}_{\lambda}^{p / 2-1}}{2^{p / 2} \Gamma(p / 2)} \frac{n K^{\operatorname{tet}}(\lambda)-n K(\lambda)}{1-F_{p}\left(n K^{\operatorname{tet}}(\lambda)\right)}$ for some $\bar{u}_{\lambda}$ between $n K(\lambda)$ and $n K^{\text {tet }}(\lambda)$. For the second term on the right hand side of (18), let

$$
r_{2 n}(\lambda)=\frac{c_{n}}{n} K(\lambda)^{\frac{p}{2}} e^{-\frac{n K(\lambda)}{2}}\left[\frac{G(\sqrt{K(\lambda)})-1}{K(\lambda)}\right]\left(1-F_{p}\left(n K^{\mathrm{tet}}(\lambda)\right)\right)^{-1}
$$

Thus, letting

$$
r_{n}(\lambda)=r_{1 n}(\lambda)+r_{2 n}(\lambda)
$$

(18) can be written as

$$
1-F_{p}(n \xi(K(\lambda)))=\left\{1-F_{p}\left(n K^{\mathrm{tet}}(\lambda)\right)\right\}\left(1+r_{n}(\lambda)\right)\left(1+O\left(n^{-1}\right)\right) .
$$


Let $R_{1 n}(\lambda), R_{2 n}(\lambda)$, and $R_{n}(\lambda)$ be the sample counterparts of $r_{1 n}(\lambda), r_{2 n}(\lambda)$, and $r_{n}(\lambda)$, respectively, obtained by replacing $K^{\text {tet }}(\lambda)$ with $\hat{K}^{\text {tet }}(\lambda)$. It remains to characterize the stochastic order of $R_{n}(\lambda)$. Note that the $\chi_{p}^{2}$ distribution satisfies

$$
\operatorname{Pr}\left\{\chi_{p}^{2} \geq u\right\}=1-F_{p}(u) \geq C e^{-u / 2} u^{p / 2-1},
$$

for some constant $C>0$.

For $R_{1 n}(\lambda)$, by using (20), we have

$$
\left|R_{1 n}(\lambda)\right| \leq O_{p}(1)\left\{n \hat{K}^{\mathrm{tet}}(\lambda)-n K(\lambda)\right\}=O_{p}\left(n^{1 / 2}|\lambda|^{2}\right)
$$

where $\hat{K}^{\text {tet }}(\lambda)=\log \left(\sum_{i=1}^{n} \hat{\pi}_{i} e^{\lambda^{\prime} g\left(X_{i}, 0\right)}\right)$ is the sample counterpart of $K^{\text {tet }}(\lambda)$, and the equality follows from (8) and $\sum_{i=1}^{n} \hat{\pi}_{i} g\left(X_{i}, 0\right) g\left(X_{i}, 0\right)^{\prime}-\mathbb{E}\left[g(X, 0) g(X, 0)^{\prime}\right]=O_{p}\left(n^{1 / 2}\right)$.

We now consider $R_{2 n}(\lambda)$. By the definition of $G(u)=1+u^{2} k(u)$ for some $k(u)$ bounded over $u \in(0, \varepsilon),\left[\frac{G(\sqrt{K(\lambda)})-1}{K(\lambda)}\right]$ is also bounded. Thus, by using $(20)$ and the definition of $c_{n}=$ $n^{p / 2} /\left(2^{p / 2-1} \Gamma(p / 2)\right)$, we have

$$
\left|R_{2 n}(\lambda)\right| \leq O_{p}(1) \hat{K}^{\mathrm{tet}}(\lambda)=O_{p}\left(|\lambda|^{2}\right)
$$

Combining these results, we obtain the conclusion $R_{n}(\lambda)=O_{p}\left(n^{1 / 2}|\lambda|^{2}\right)$.

\section{A.4 Proof of Theorem 4}

\section{Proof of Part (i)}

Let $\Omega=\mathbb{E}\left[g\left(X, \theta_{0}\right) g\left(X, \theta_{0}\right)^{\prime}\right]$ and $M=\Omega^{-1 / 2} \mathbb{E}\left[\partial g\left(X, \theta_{0}\right) / \partial \theta_{1}^{\prime}\right]$. By the spectral decomposition of the idempotent matrix (Czellar and Ronchetti, 2010), there exists a matrix $C=\left[C_{1}: C_{2}\right]$ such that

$$
M\left(M^{\prime} M\right)^{-1} M^{\prime}=C\left[\begin{array}{cc}
I_{p_{1}} & 0 \\
0 & 0_{p_{2} \times p_{2}}
\end{array}\right] C^{\prime},
$$

and $C^{\prime} C=C C^{\prime}=I_{p}$ with $p=p_{1}+p_{2}$. Based on Newey and Smith (2004, p. 240), we can see that $\sqrt{n} \tilde{\lambda}$ is asymptotically equivalent to $\sqrt{n} \Omega^{-1 / 2} C_{2} \tilde{\gamma}$, where $\tilde{\gamma}$ solves

$$
\sum_{i=1}^{n} e^{\tilde{\gamma}^{\prime} \tilde{g}\left(X_{i}, \theta_{0}\right)} \tilde{g}\left(X_{i}, \theta_{0}\right)=0
$$

where

$$
\tilde{g}\left(X, \theta_{0}\right)=C_{2}^{\prime} \Omega^{-1 / 2} g\left(X, \theta_{0}\right) .
$$


The saddlepoint density of $\tilde{\gamma}$ is given by (6) with replacement of $g\left(X_{i}, \theta_{0}\right)$ with $\tilde{g}\left(X_{i}, \theta_{0}\right)$. Let $\tilde{K}(\gamma)=K\left(\Omega^{-1 / 2} C_{2} \gamma, \tilde{\theta}_{1}\left(\Omega^{-1 / 2} C_{2} \gamma\right)\right)$. We can also see that

$$
\operatorname{Pr}\left\{n T_{n, c} \geq n t_{n, c}: F\right\}=\operatorname{Pr}\left\{2 n \tilde{K}(\tilde{\gamma}) \geq n \tilde{K}\left(\tilde{\gamma}_{\mathrm{o}}\right): F\right\}\left(1+O\left(e^{-n \epsilon}\right)\right)
$$

for any $\epsilon>0$ small enough. Then the conclusion follows as in the proof of Theorem 2 by replacing $h^{\lambda}(y)=\log \mathbb{E}\left[e^{y^{\prime} g(X, 0)}\right]$ with $\log \mathbb{E}\left[e^{y^{\prime} \tilde{g}\left(X, \theta_{0}\right)}\right]$.

\section{Proof of Part (ii)}

Using the spectral decomposition of idempotent matrix adopted in the proof of (i), we can show that $n K^{\text {tet }}\left(\lambda, \tilde{\theta}_{1}^{\text {tet }}(\lambda)\right)-n K\left(\lambda, \tilde{\theta}_{1}(\lambda)\right)=O\left(n^{1 / 2}|\lambda|^{2}\right)$. Therefore, (ii) follows by using the same arguments adopted for the proof of Theorem 3 .

\section{A.5 Proof of Theorem 5}

The proof is similar to that of Theorem 4 . 


\section{B Figures for Section 4}

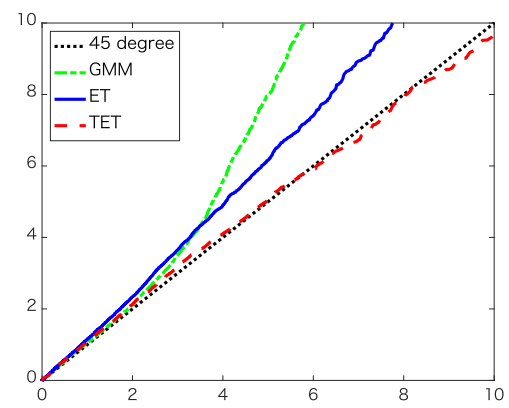

(a) Normal, $\delta^{2}=50$

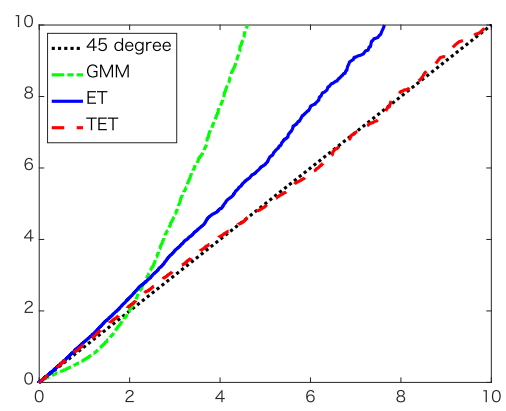

(b) Normal, $\delta^{2}=10$

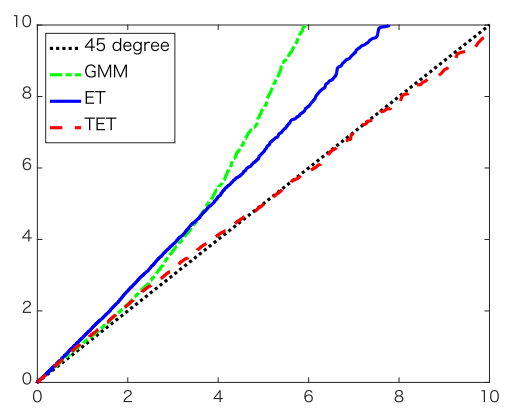

(c) $\chi_{3}^{2}, \delta^{2}=50$

Figure 2: q-q plots for $H_{0}: \theta_{2}=0$ with $n=40$ : (a) Normal, $\delta^{2}=50$, (b) Normal, $\delta^{2}=10$, and (c) $\chi_{3}^{2}, \delta^{2}=50$.

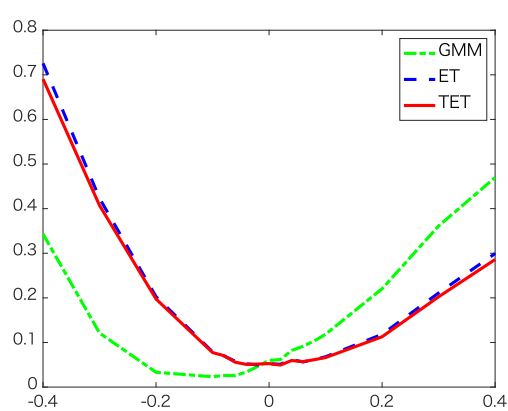

(a) Normal, $\delta^{2}=50$

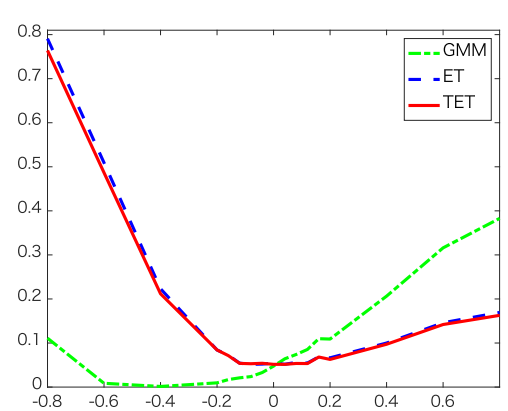

(b) Normal, $\delta^{2}=10$

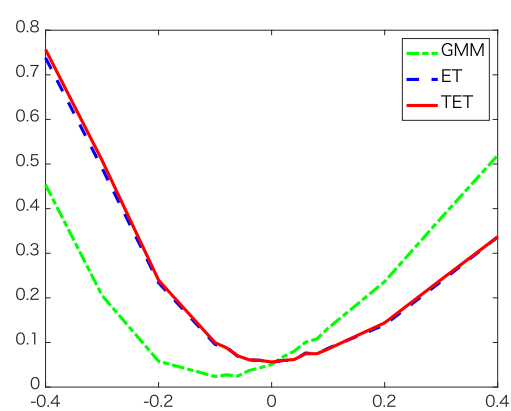

(c) $\chi_{3}^{2}, \delta^{2}=50$

Figure 3: Calibrated powers for $H_{0}: \theta_{2}=0$ with $n=40$ : (a) Normal, $\delta^{2}=50$, (b) Normal, $\delta^{2}=10$, and (c) $\chi_{3}^{2}, \delta^{2}=50$.

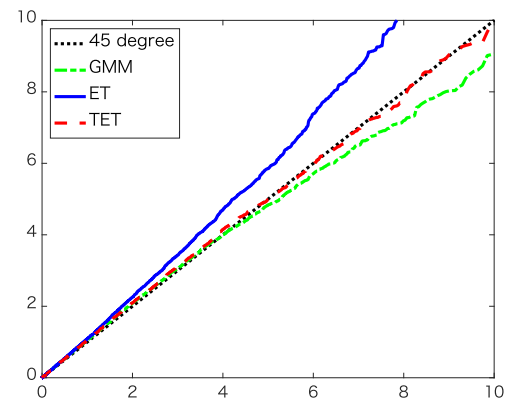

(a) Normal, $\delta^{2}=50$

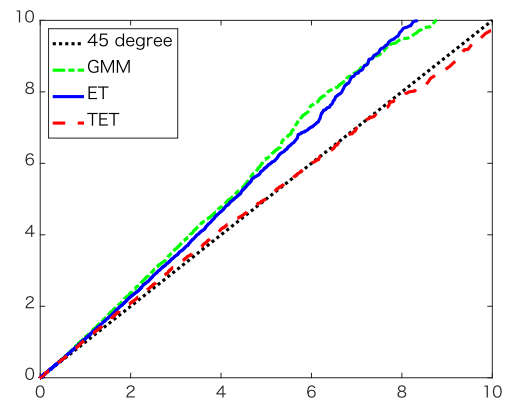

(b) Normal, $\delta^{2}=10$

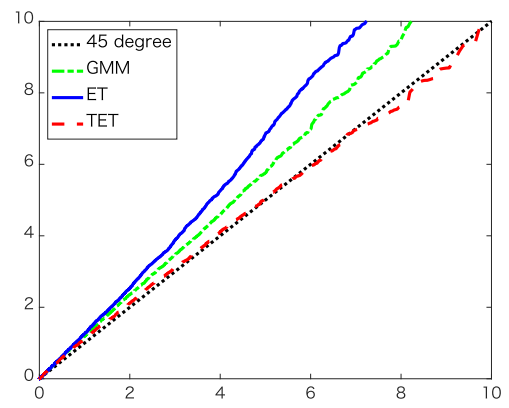

(c) Normal, $\delta^{2}=50$, heteroskedastic

Figure 4: q-q plots for overidentification with $n=40$ : (a) Normal, $\delta^{2}=50$, (b) Normal, $\delta^{2}=10$, and (c) Normal, $\delta^{2}=50$, heteroskedastic. 


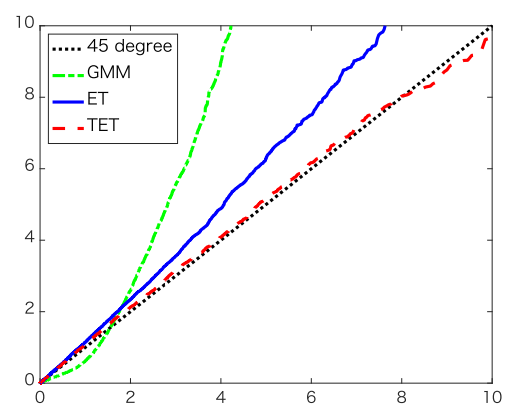

(a) Normal, $\delta^{2}=5$

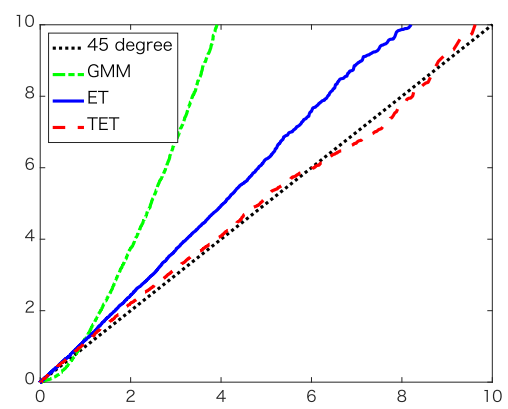

(b) Normal, $\delta^{2}=1$

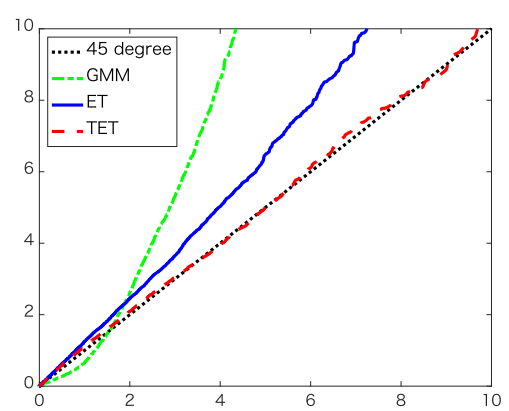

(c) $\chi_{3}^{2}, \delta^{2}=5$

Figure 5: q-q plots for $H_{0}: \theta_{2}=0$ with $n=40$ : (a) Normal, $\delta^{2}=5$, (b) Normal, $\delta^{2}=1$, and (c) $\chi_{3}^{2}, \delta^{2}=5$.

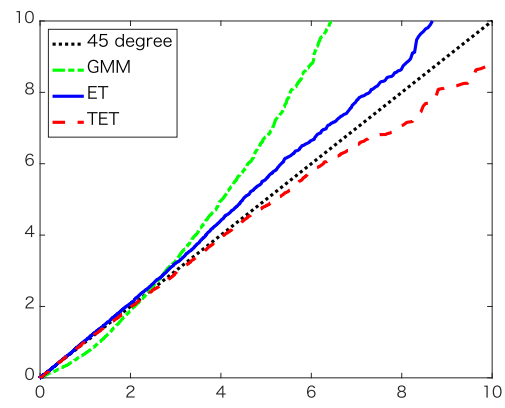

(a) Normal, $\delta^{2}=5$

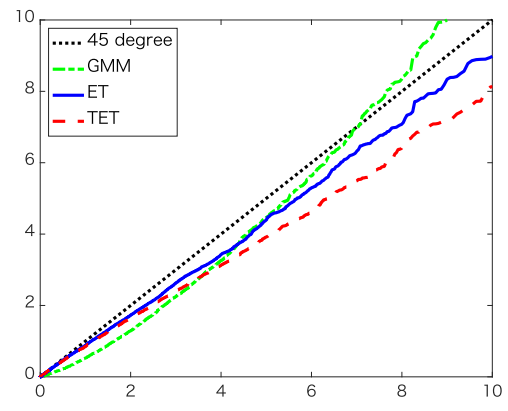

(b) Normal, $\delta^{2}=1$

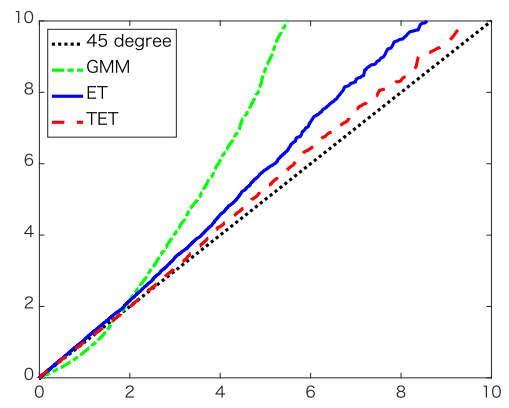

(c) $\chi_{3}^{2}, \delta^{2}=5$

Figure 6: q-q plots for $H_{0}: \theta_{1}=0$ with $n=40$ : (a) Normal, $\delta^{2}=5$, (b) Normal, $\delta^{2}=1$, and (c) $\chi_{3}^{2}, \delta^{2}=5$. 


\section{References}

[1] Almudevar, A., Field, C. and J. Robinson (2000) The density of multivariate M-estimates, Annals of Statistics, 28, 275-297.

[2] Andrews, D. W. K. and J. H. Stock (2007) Inference with weak instruments, in Advances in Economics and Econometrics, Theory and Applications, Ninth World Congress, vol. 3, Blundell, R., Newey, W. and T. Persson (eds.), Cambridge University Press, pp. 122-173.

[3] Baggerly, K. A. (1998) Empirical likelihood as a goodness-of-fit measure, Biometrika, 85, $535-547$.

[4] Brown, B. W. and W. K. Newey (1998) Efficient semiparametric estimation of expectations, Econometrica, 66, 453-464.

[5] Czellar, V. and E. Ronchetti (2010) Accurate and robust tests for indirect inference, Biometrika, 97, 621-630.

[6] Daniels, H. E. and G. A. Young (1991) Saddlepoint approximation for the studentized mean, with an application to the bootstrap, Biometrika, 78, 169-179.

[7] Efron, B. (1981) Nonparametric standard errors and confidence intervals, Canadian Journal of Statistics, 9, 139-172.

[8] Efron, B. (1982) Transformation theory: How normal is a family of distributions?, Annals of Statistics, 10, 323-339.

[9] DiCiccio, T. J., Hall, P. and J. Romano (1991) Empirical likelihood is Bartlett-correctable, Annals of Statistics, 19, 1053-1061.

[10] Field, C. A. (1982) Small sample asymptotic expansions for multivariate M-estimates, Annals of Statistics, 10, 672-689.

[11] Huber, P. J. and E. M. Ronchetti (2009) Robust Statistics, 2nd edition, Wiley.

[12] Imbens, G. W., Spady, R. H. and P. Johnson (1998) Information theoretic approaches to inference in moment condition models, Econometrica, 66, 333-357.

[13] Jensen, J. L. (1995) Saddlepoint Approximations, Oxford University Press.

[14] Jensen, J. L. and A. T. A. Wood (1998) Large deviation and other results for minimum contrast estimators, Annals of the Institute of Mathematical Statistics, 50, 673-695. 
[15] Jing, B. Y. and J. Robinson (1994) Saddlepoint approximations for marginal and conditional probabilities of transformed variables, Annals of Statistics, 22, 1115-1132.

[16] Jing, B. Y., Shao, Q. M. and W. Zhou (2004) Saddlepoint approximation for student's t-statistic with no moment conditions, Annals of Statistics, 32, 2697-2711.

[17] Jing, B. Y. and A. T. A. Wood (1996) Exponential empirical likelihood is not Bartlett correctable, Annals of Statistics, 24, 365-369.

[18] Kitamura, Y. (2007) Empirical likelihood methods in econometrics: theory and practice, in Blundell, R., Newey, W. K. and T. Persson (eds.), Advances in Economics and Econometrics, vol. III, 174-237.

[19] Kitamura, Y. and M. Stutzer (1997) An information-theoretic alternative to generalized method of moments estimation, Econometrica, 65, 861-874.

[20] Kolassa, J. and J. Robinson (2011) Saddlepoint approximations for likelihood ratio like statistics with applications to permutation tests, Annals of Statistics, 39, 3357-3368.

[21] Ma, Y. and E. Ronchetti (2011) Saddlepoint test in measurement error models, Journal of the American Statistical Association, 106, 147-156.

[22] Newey, W. K. and R. J. Smith (2004) Higher order properties of GMM and generalized empirical likelihood estimators, Econometrica, 72, 219-255.

[23] Owen, A. B. (1988) Empirical likelihood ratio confidence intervals for a single functional, Biometrika, 75, 237-249.

[24] Owen, A. B. (2001) Empirical Likelihood, New York, Chapman and Hall.

[25] Phillips, P. C. B. (1980) The exact distribution of instrumental variable estimators in an equation containing $n+1$ endogenous variables, Econometrica, 48, 861-878.

[26] Phillips, P. C. B. (1989) Partially identified econometric models, Econometric Theory, 5, $181-240$.

[27] Robinson, J., Ronchetti, E. and G. A. Young (2003) Saddlepoint approximations and tests based on multivariate M-estimates, Annals of Statistics, 31, 1154-1169.

[28] Skovgaard, I. M. (1990) On the density of minimum contrast estimators, Annals of Statistics, 18, 779-789. 
[29] Staiger, D. and J. H. Stock (1997) Instrumental variables regression with weak instruments, Econometrica, 65, 557-586.

[30] Tingley, M. A. and C. A. Field (1990) Small-sample confidence intervals, Journal of the American Statistical Association, 85, 427-434. 